\title{
Accurate Stellar Population Studies from Multiband Photometric Observations
}

\section{Citation}

Romaniello, Martino, Nino Panagia, Salvatore Scuderi, and Robert P. Kirshner. 2002. "Accurate Stellar Population Studies from Multiband Photometric Observations." The Astronomical Journal 123 (2): 915-40. https://doi.org/10.1086/338430.

\section{Permanent link}

http://nrs.harvard.edu/urn-3:HUL.InstRepos:41417339

\section{Terms of Use}

This article was downloaded from Harvard University's DASH repository, and is made available under the terms and conditions applicable to Other Posted Material, as set forth at http:// nrs.harvard.edu/urn-3:HUL.InstRepos:dash.current.terms-of-use\#LAA

\section{Share Your Story}

The Harvard community has made this article openly available.

Please share how this access benefits you. Submit a story.

Accessibility 


\title{
ACCURATE STELLAR POPULATION STUDIES FROM MULTIBAND PHOTOMETRIC OBSERVATIONS ${ }^{1}$
}

\author{
Martino Romaniello \\ European Southern Observatory, Karl-Schwarzschild-Strasse 2, D-85748 Garching bei München, Germany; mromanie@eso.org \\ Nino PANAGia ${ }^{2}$ \\ Space Telescope Science Institute, 3700 San Martin Drive, Baltimore, MD 21218; panagia@stsci.edu \\ Salvatore ScUderi \\ Osservatorio Astrofisico di Catania, viale A. Doria 6, I-95125 Catania, Italy; scuderi@sunct.ct.astro.it \\ AND \\ ROBERT P. KIRSHNER \\ Harvard-Smithsonian Center for Astrophysics, 60 Garden Street, Cambridge, MA 02138; kirshner@cfa.harvard.edu \\ Received 2001 March 8; accepted 2001 October 31
}

\begin{abstract}
We present a new technique based on multiband near-ultraviolet and optical photometry to measure both the stellar intrinsic properties, i.e., luminosity and effective temperature, and the interstellar dust extinction along the line of sight to hundreds of stars per square arcminute. The yield is twofold. On the one hand, the resulting reddening map has a very high spatial resolution, of the order of a few arcseconds, and can be quite effectively used in regions where the interstellar material is patchy, thus producing considerable differential extinction on small angular scales. On the other hand, combining the photometric information over a wide baseline in wavelength provides an accurate determination of temperature and luminosity for thousands of stars. As a test case, we present the results for the region around SN 1987A in the Large Magellanic Cloud imaged with the Wide Field Planetary Camera 2 on board the Hubble Space Telescope.

Key words: dust, extinction — galaxies: individual (Large Magellanic Cloud) -

stars: fundamental parameters — supernovae: individual (SN 1987A)
\end{abstract}

\section{INTRODUCTION}

Stars provide most of the light we detect in the universe, and the entire chemical and dynamical evolution of galaxies is strongly influenced by the formation of many generations of stars. The most massive ones enrich the surrounding medium of highly processed material and supply vast amounts of kinetic energy (both with their winds and even more with their ultimate explosions as Type II supernovae). Lower mass stars constitute one of the main sources of crucial elements, such as carbon and nitrogen (through stellar winds first and planetary nebula ejection later) in their individual evolution and may be the main suppliers of the iron group elements if in binary systems they succeed to merge and explode as Type Ia supernovae.

A major problem that one encounters almost ubiquitously when studying stars is the effect that interstellar dust has on the observed spectral distribution of their light. A precise measurement of the interstellar reddening along the line of sight to the stars under study is the first step, a crucial one, toward determining the intrinsic properties of the stellar populations. All-sky reddening maps, such as the one derived from dust temperature by Schlegel, Finkbeiner, \& Davis (1998), may not be satisfactory for detailed stellar populations studies. For example, it cannot be used in the direction of nearby galaxies, such as the Magellanic Clouds and M31. Also, the derived value of the dust column density

\footnotetext{
${ }^{1}$ Based on observations with the NASA/ESA Hubble Space Telescope, obtained at the Space Telescope Science Institute, which is operated by AURA, Inc., under NASA contract NAS 5-26555.

${ }^{2}$ On assignment from the Research and Scientific Support Department of ESA.
}

is extremely sensitive to dust temperature and, in any case, not accurate for high values of extinction $\left(A_{V}>0.5 \mathrm{mag}\right.$; Arce \& Goodman 1999). Moreover, the angular resolution of the map is pretty coarse $\left(\sim 6^{\prime}\right.$, compared with a few arcseconds for the method presented here), and thus it does not allow us to cope effectively with the patchy structure typical of the interstellar medium.

It is certainly preferable to determine the amount of dust in front of the stars from the light coming from the stars themselves. While spectroscopic data may certainly provide an accurate determination of the reddening and the stellar parameters, the multiplexing capabilities of current spectrographs are limited, at best, to a few tens of (relatively bright) objects per telescope pointing. The method we describe in this paper overcomes this limitation by using only imaging and thus providing measurements of both the stellar parameters and the reddening along the line of sight to thousands of stars per pointing. The gain in terms of statistics is obviously tremendous. As a consequence, the spatial resolution of the resulting extinction map is extremely high, with an $E(B-V)$ measurement every few arcseconds, even for moderately dense star fields. While here we test this newly developed technique on Hubble Space Telescope (HST) Wide Field Planetary Camera 2 (WFPC2) observations, the method can be applied to data obtained with any instrument. The quality of the final result, however, will obviously depend on the quality of the input photometry.

The whole purpose of this paper is to describe a method to retrieve the stellar parameters from this kind of data by fitting model atmospheres to the observed fluxes. In $\S 2$ we describe the data set considered and the reduction techniques adopted. In $\S 3$ the limitations of the classical approach based on color-magnitude diagrams are discussed, and the 
method we have developed to overcome them via a multiband fit to the observed spectra is presented in $\S 4$. The results of this procedure are discussed in $\S 5$, while $\S 6$ deals with the effects that the gravity and metallicity of the input model spectra have on the derived parameters. Finally, the spectral coverage needed to recover both $T_{\text {eff }}$ and $E(B-V)$ is explored in $\S 7$, and the role of random and systematic errors is assessed in $\S 8$.

The broadband colors in selected WFPC2 filters and the corresponding extinction coefficients, which are needed for any application of our procedure, are provided in Appendix A.

\section{OBSERVATIONS AND DATA REDUCTION}

Our method was developed and tested using HST WFPC2 multiband observations. In particular, we have taken advantage of the wealth of information provided by the repeated observations of SN 1987A and the field surrounding it in the Large Magellanic Cloud (LMC). Since 1994, SN 1987A was imaged with the WFPC2 every year as part of the Supernova Intensive Study project (SINS; principal investigator, Robert P. Kirshner). The log of the observations we have used is reported in Table 1. The WFPC2 filters do not exactly match any of the ground-based photometric systems, and the closest analog to the passbands we have used is reported in the last column of this table. The observations cover an almost circular portion of the sky centered on SN 1987A, with a radius of $130^{\prime \prime}$, corresponding to roughly $32 \mathrm{pc}$ at the distance of the LMC $(51.4 \mathrm{kpc}$; Panagia 1999; Romaniello et al. 2000). The true color image derived from our data is shown in Figure 1.

The observations were processed through the standard Post Observation Data Processing System pipeline for bias removal and flat fielding. In all the cases the available images for each filter were combined to remove cosmic-ray events. The long baseline they cover in wavelength (more than a factor of 4 , extending from $\sim 2300$ to $\sim 9600 \AA$ ), together with the unprecedented spatial resolution of WFPC2 ( 0 ". 1 , i.e., roughly $0.03 \mathrm{pc}$ at the distance of the LMC), make this data set a unique and ideal one to investigate the problem of disentangling the effects of reddening from those of different effective temperatures for stars of different spectral type.

The plate scale of the camera is 0.045 and 0.099 pixel $^{-1}$ in the PC and in the three WF chips, respectively. We performed aperture photometry following the prescriptions by
Gilmozzi (1990) as refined by Romaniello (1998), i.e., measuring the flux in a circular aperture of radius 2 pixels and the sky background value in an annulus of internal radius 3 pixels and width 2 pixels. Aperture photometry is perfectly adequate for our study because crowding is never a problem in our images. Actually, the average separation of stars from each other is about 1"3 (i.e., 29 pixels in the PC chip and $\sim 13$ pixels in the WF ones), which is much larger than the WFPC2 point-spread function (PSF) width. We have used the IRAF SYNPHOT synthetic photometry package to compute the theoretical data numbers to compare with the observed ones. The WFPC2 calibration and, consequently, the SYNPHOT results are typically accurate to within $\pm 5 \%$ (Whitmore 1995; Bagget et al. 1997).

We identify the stars in the deepest frame (F555W, i.e., $V$ band-like filter) and then measure their magnitudes in all the other filters. The total number of stars identified in this way is 21,955 . If a star is not detected in a band, then its magnitude in that band is substituted by a $3 \sigma$ upper limit. For about 12,340 of them, i.e., $56 \%$, the photometric accuracy is better than $0.1 \mathrm{mag}$ in the $\mathrm{F} 555 \mathrm{~W}, \mathrm{~F} 675 \mathrm{~W}$, and F814W filters. The number of stars with accuracy better than 0.1 mag drops to $6825(31 \%)$ in the F439W band, and only $786(3.6 \%)$ stars have an uncertainty in the F255W filter smaller than $0.2 \mathrm{mag}$.

The brightest stars in each CCD chip are saturated (i.e., most stars brighter than 17.5 in the F555W passband). We recover their photometry either by fitting the unsaturated wings of the PSF for moderately saturated stars, i.e., those with no saturation outside the central 2 pixel radius, or by following the method developed by Gilliland (1994) for heavily saturated stars. In most cases, the achieved photometric accuracy is better than 0.05 mag. Full description of the methods used can be found in Romaniello (1998). As a sanity check we have compared our photometry in the F439W and F555W bands with the ground-based one in $B$ and $V$ (28 stars brighter than $V \simeq 20$ within $30^{\prime \prime}$ from SN 1987A; Walker \& Suntzeff 1990) and found an overall excellent agreement, i.e., rms deviations less than $0.05 \mathrm{mag}$ in both filters, except for the obvious cases of a few stars that appear pointlike in ground-based images but are resolved into more than one object on the WFPC2 frames. This result confirms the accuracy of our photometric measurements and the quality of the WFPC2 calibration.

We shall see in $\S 4$ that a good photometric quality in different bands is needed to recover $E(B-V)$ and stellar temperature. To select stars with a good overall photometry, we

TABLE 1

Log of the Observations Centered on SN 1987A

\begin{tabular}{|c|c|c|c|c|}
\hline \multirow[b]{2}{*}{ Filter Name } & \multicolumn{3}{|c|}{ Exposure Time (s) } & \multirow[b]{2}{*}{ Comments } \\
\hline & 1994 September ${ }^{\mathrm{a}}$ & 1996 February $^{\mathrm{b}}$ & 1997 July $^{\mathrm{c}}$ & \\
\hline F255W ............... & $2 \times 900$ & $1100+1400$ & $2 \times 1300$ & $U V$ filter \\
\hline F336W ............ & $2 \times 600$ & $2 \times 600$ & $2 \times 800$ & $U$ Filter \\
\hline F439W ............ & $2 \times 400$ & $350+600$ & $2 \times 400$ & $B$ filter \\
\hline F555W ............ & $2 \times 300$ & $2 \times 300$ & $2 \times 300$ & $V$ filter \\
\hline F675W ............ & $2 \times 300$ & $2 \times 300$ & $2 \times 300$ & $R$ filter \\
\hline F814W ............ & $2 \times 300$ & $2 \times 300$ & $2 \times 400$ & $I$ filter \\
\hline
\end{tabular}

a 1994 September 24, proposal number 5753.

b 1996 February 6, proposal number 6020.

c 1997 July 10, proposal number 6437. 


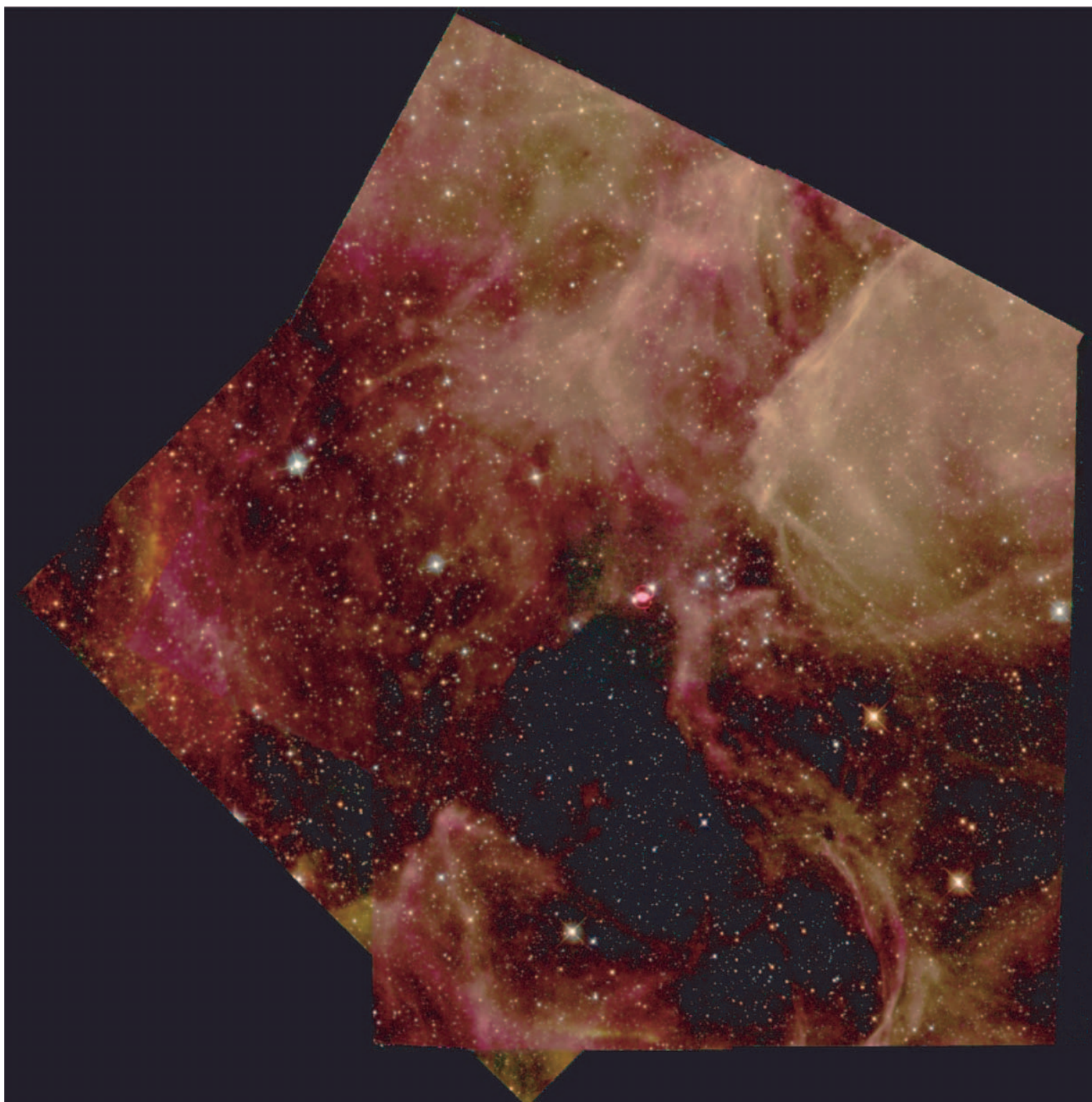

FIG. 1.-Field centered on SN 1987A (about 130" radius) as observed in the combination of the $B, V$, and $I$ broad bands plus the [O III] and $\mathrm{H} \alpha$ narrowband images.

define the mean error in five bands $\left(\bar{\delta}_{5}\right)$ (i.e., excluding the UV F255W band) as

$$
\bar{\delta}_{5}=\sqrt{\frac{\delta_{\mathrm{F} 336 \mathrm{~W}}^{2}+\delta_{\mathrm{F} 439 \mathrm{~W}}^{2}+\delta_{\mathrm{F} 555 \mathrm{~W}}^{2}+\delta_{\mathrm{F} 675 \mathrm{~W}}^{2}+\delta_{\mathrm{F} 814 \mathrm{~W}}^{2}}{5}} .
$$

In addition to the main field described above, we have also used a second set of WFPC 2 images pointing $8^{\prime}$ southeast of SN 1987A. As shown in Table 2 in this case we use only four filters, and the F336W and F439W passbands are substituted with their broader counterparts, F300W and F450W, respectively. Being broader, the latter are more efficient and allowed us to save precious telescope time, always a crucial issue, without losing any vital information (see $\S 7$ ).

\section{COLOR-MAGNITUDE DIAGRAMS}

As it can be seen in Figure 1 SN 1987A and its rings are right at the center of the field imaged with WFPC2 and belong to a loose cluster of blue stars (see Panagia et al. 2000). Also, the interstellar medium, as traced by the $\mathrm{H} \alpha$ emission coded in red in Figure 1, is far from being spatially uniform and its distribution varies on scales of a few arcseconds (let us recall here that $1^{\prime \prime}$ corresponds to roughly 0.25 $\mathrm{pc}$ at the distance of the LMC). Since the spatial distribution

TABLE 2

Log of the ObServations of the Control Field FOR SN 1987A

\begin{tabular}{lrl}
\hline \hline Filter Name & $\begin{array}{c}\text { Exposure Time } \\
(\mathrm{s})\end{array}$ & \multicolumn{1}{c}{ Comments } \\
\hline F300W .......... & $600+1200$ & Wide $U$ filter \\
F450W ........... & $50+200$ & Wide $B$ filter \\
F675W ......... & $50+200$ & $R$ filter \\
F814W .......... & $140+400$ & $I$ filter \\
\hline
\end{tabular}

Note._-1997 January 1, proposal number 6437. 
of dust usually follows the one of gas (see, for example, Mathis 1990), one can expect the interstellar reddening to vary on similar, if not even smaller, scales. This is further confirmed by an inspection of Figure 2, where we show the color-magnitude diagrams (CMDs) for different combinations of bands.

The black dots in each CMD in Figure 2 are the 6695 stars with $\bar{\delta}_{5}<0.1 \mathrm{mag}$, where $\bar{\delta}_{5}$ is the mean error defined in equation (1). The error threshold of $\bar{\delta}_{5}<0.1 \mathrm{mag}$ is reflected by a magnitude threshold at $m_{\mathrm{F} 555 \mathrm{~W}} \simeq 23$. We estimate that the completeness at this magnitude limit is very close to $100 \%$ because the density of detected stars down to $23 \mathrm{~d}$ magnitude is rather low, i.e., about 1 star per $4.6 \operatorname{arcsec}^{2}$ area (Panagia et al. 2000).

It is apparent that, despite the high quality of the measurements (internal uncertainties less than $0.1 \mathrm{mag}$; see also Fig. 3), the various features of the CMDs, such as the zeroage main sequence for the early-type stars and the red giant clump for the more evolved populations, are rather "fuzzy" and not sharply defined. Although this is due in part to the presence of several stellar populations projected on each other (Panagia et al. 2000), most of the problem arises because reddening is not quite uniform over the field, thus causing the points of otherwise identical stars to fall in appreciably different locations of the CMDs.

\section{FITTING PROCEDURE}

The large number of bands available in our data set provides a sort of wide-band spectroscopy, which defines the continuum spectral distribution of each star quite well. Of course, the resolution is very low, of the order of $\lambda / \Delta \lambda \simeq 7$, but still, important features, such as the Balmer jump between the HST F336W and F439W bands, are clearly visible. Four examples of "wide-band spectra" are shown in Figure 4 (the flux $F_{\lambda}$ is expressed in ergs $\mathrm{cm}^{-2} \mathrm{~s}^{-1} \AA^{-1}$ ), and their observed magnitudes are listed in Table 3 . The whole purpose of this paper is to describe a method to retrieve the stellar parameters from this kind of data by fitting model atmospheres to the observed fluxes.

The shape of the observed spectrum is mostly determined by the effective temperature of the star and by the amount of dust along the line of sight. The other quantity of interest, namely, the solid angle subtended by the star (or, correspondingly, its angular radius) is obtained by a straight normalization of the spectral distribution.

The general idea of our method is to recover simultaneously the extinction along the line of sight and the effective temperature of a star by fitting the observed magnitudes to the ones computed using stellar atmosphere models reddened by various amounts of $E(B-V)$. The angular radius, then, is directly obtained from the ratio of the observed flux, dereddened with the best-fitting $E(B-V)$ value, to the one at the surface of the star, as given by the best-fitting model atmosphere. Thus, the two fundamental ingredients that go into the fit are the stellar atmosphere models and the reddening law: we have adopted the models by Bessel, Castelli, \& Plez (1998) and the reddening law by Scuderi et al. (1996).

We have chosen the models by Bessel et al. (1998), as they provide a well-tested, homogeneous set covering the tem-
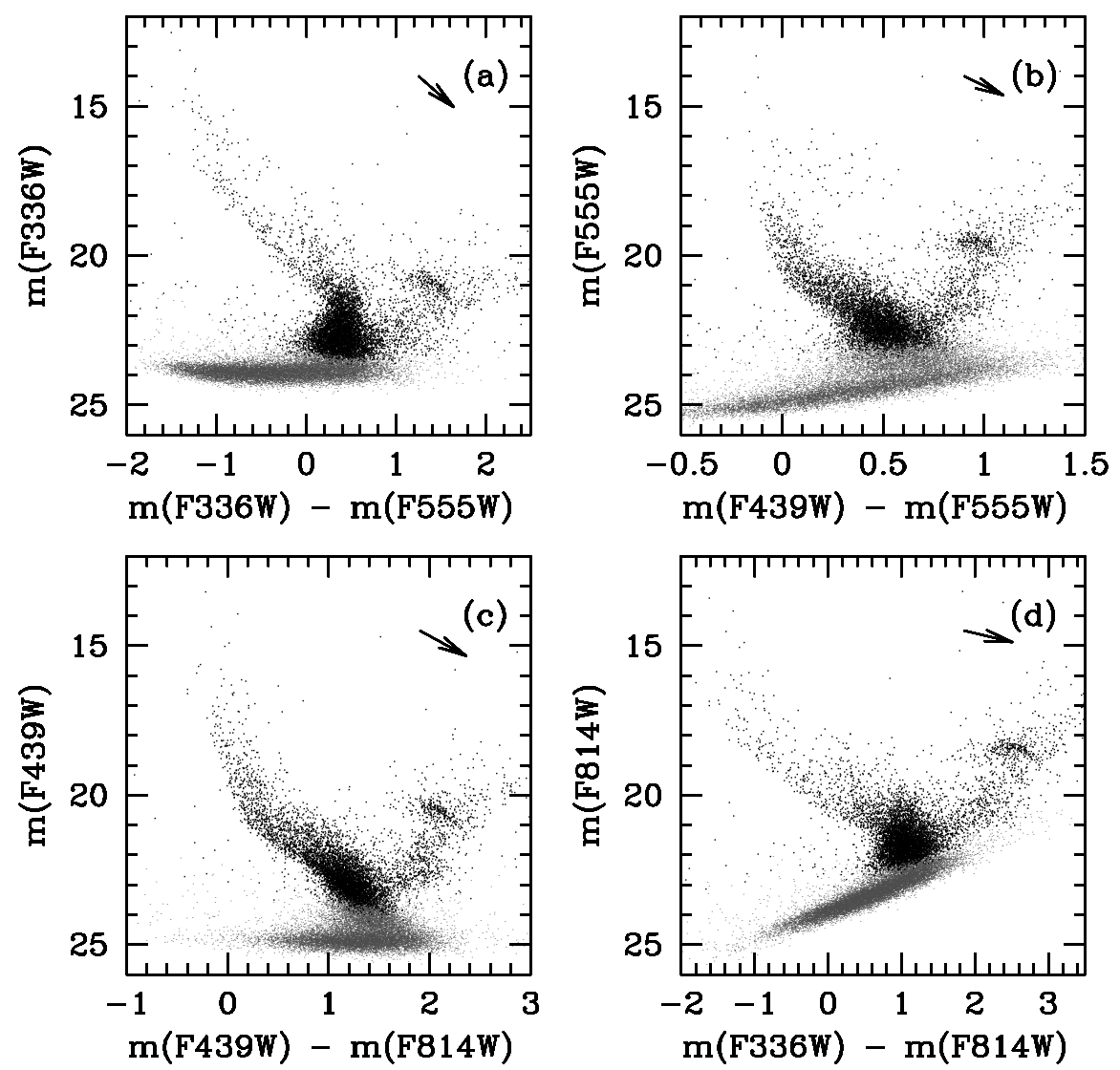

FIG. 2.- Color-magnitude diagrams for four combinations of filters, showing stars with average errors, as defined in eq. (1), $\bar{\delta}_{5}>0.1$ (gray dots) and the 6695 stars with $\bar{\delta}_{5}<0.1$ (black dots). The reddening vector corresponding to $E(B-V)=0.2$ is shown in each panel. 


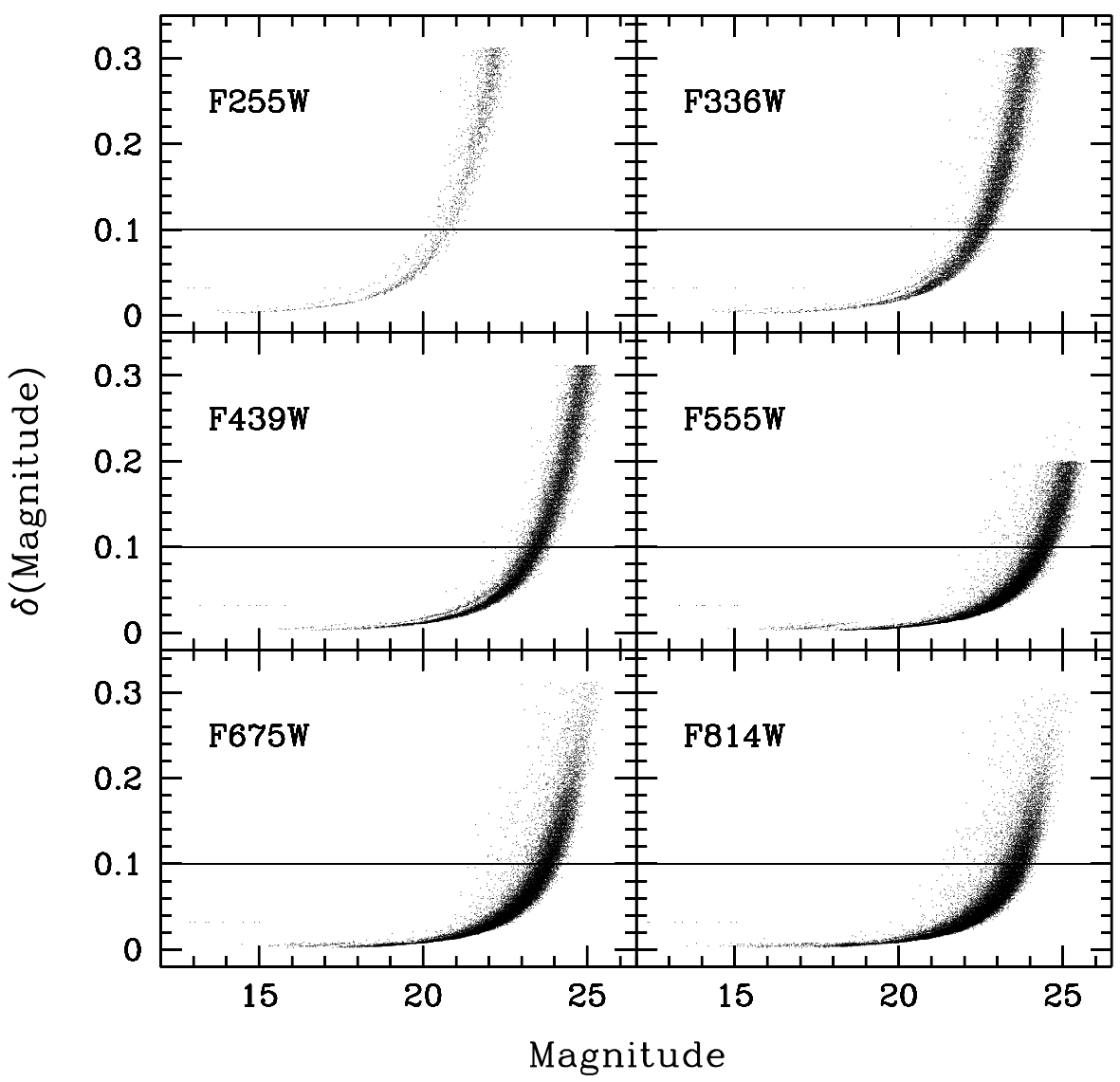

FIG. 3.-Photometric error as a function of magnitude for the six broadband filters around SN 1987A. The 0.1 mag error level is indicated.

perature interval between 3500 and $50,000 \mathrm{~K}$ in the wavelength range between $90 \AA$ and $160 \mu \mathrm{m}$. They are computed with an updated version of the ATLAS9 code used for the Kurucz (1993) models with no allowance for convective

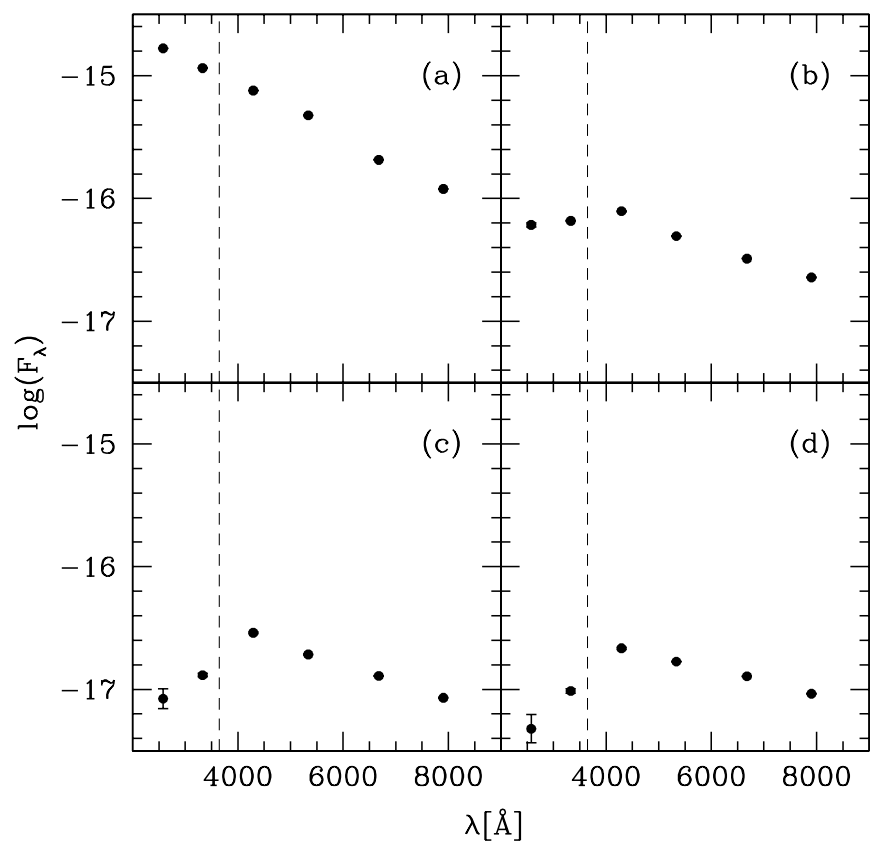

FIG. 4.-Four examples of the spectral resolution of the "wide-band spectroscopy," showing the position of the Balmer jump at 3646 A (dashed line). The magnitudes and errors of these four stars are given in Table 3. overshooting, as discussed by Castelli, Gratton, \& Kurucz (1997a, 1997b).

We have used the IRAF task SYNPHOT to compute the theoretical magnitudes at the surface of the star for the grid of temperatures provided by Bessel et al. (1998) by convolving the model spectra with the HST WFPC2 response curves. Once the convolution is performed, we use a spline interpolation to get a temperature grid with a logarithmic step of 0.005 , i.e., $1 \%$. We have used model atmospheres with $\log g=4.5$ (cgs units), which is the typical value for main-sequence stars, and $Z=0.3 Z_{\odot}$, the mean expected metallicity of the LMC. In $\S 6$ we will analyze and discuss the effects of these two parameters on the fit, and we will see that neither of them affects the values of the derived parameters appreciably.

To faithfully model the data, we have computed separately the expected counts that a given spectrum produces on each of the four WFPC2 chip detectors. Also, the throughput variation between decontaminations of the camera (Bagget et al. 1996) was taken into account by specifying the Julian date of the observation (the keyword "cont" in SYNPHOT). Details of this procedure can be found in Romaniello (1998), while model magnitudes for solar and one-third solar metallicity are listed here in Tables 6 and 7 , respectively.

The other input ingredient is the reddening law. This is a combination of foreground extinction due to dust in the Milky Way and internal extinction in the LMC. We have taken the first component to be the same for all the stars, with $E(B-V)_{\mathrm{MW}}=0.05$ (see, for example, Schwering \& Israel 1991), and we have used the Galactic reddening law 
TABLE 3

Observed Magnitudes and Statistical Errors for the Stars of Figure 4

\begin{tabular}{ccccccc}
\hline \hline Panel & F255W & F336W & F439W & F555W & F675W & F814W \\
\hline$a \ldots \ldots \ldots .$. & $15.74 \pm 0.01$ & $16.13 \pm 0.01$ & $17.37 \pm 0.01$ & $17.23 \pm 0.01$ & $17.44 \pm 0.01$ & $17.48 \pm 0.01$ \\
$b \ldots \ldots \ldots$. & $19.34 \pm 0.04$ & $19.24 \pm 0.01$ & $19.83 \pm 0.01$ & $19.69 \pm 0.01$ & $19.45 \pm 0.01$ & $19.28 \pm 0.01$ \\
$c \ldots \ldots \ldots .$. & $21.50 \pm 0.20$ & $20.99 \pm 0.03$ & $20.91 \pm 0.02$ & $20.71 \pm 0.01$ & $20.45 \pm 0.01$ & $20.35 \pm 0.01$ \\
$d \ldots \ldots \ldots .$. & $22.10 \pm 0.30$ & $21.32 \pm 0.04$ & $21.23 \pm 0.02$ & $20.85 \pm 0.01$ & $20.46 \pm 0.01$ & $20.26 \pm 0.01$ \\
\hline
\end{tabular}

as compiled by Savage \& Mathis (1979). On the other hand, the amount of extinction from the LMC dust is one of the parameters of the fit. We have taken the LMC reddening law in the direction of our field to be the one determined by Scuderi et al. (1996) from a study of an HST Faint Object Spectograph (FOS) ultraviolet and optical spectrum of star 2, one of the companions to SN 1987A projected just 3".9 northwest of it. It is, of course, crucially important to use a reddening law derived in the same field under study, as it may vary considerably from place to place on quite small angular scales (see, for example, Whittet 1992). These reddening laws were used to compute the extinction coefficients in the WFPC2 passbands for every combination of input model spectrum and value of $E(B-V)$ (see Table 8).

The fitting procedure consists of two steps:

1. We first use a reddening-free color to determine which stars can be used to measure the reddening unambiguously.

2. Once this is done, we use the full information from the six bands to fit the observed spectral energy distribution for all the stars.

We will now describe these two steps.

\subsection{First Step: Reddening-free Colors}

It is a well known fact that the solution in the $E(B-V)$ $T_{\text {eff }}$ plane may not be unique for stars later than, roughly speaking, A0, and that different combinations of temperature and reddening may lead to almost indistinguishable optical and near-UV low-resolution spectra. An example is shown in Figure 5, in which we compare Bessel et al. (1998) models for three combinations of temperature and reddening: $T_{\mathrm{eff}}=5000 \mathrm{~K}$ and $E(B-V=0)$ (short-dashed line), $T_{\text {eff }}=7600 \mathrm{~K}$, reddened by $E(B-V)=0.55$ (long-dashed line), and finally $T_{\mathrm{eff}}=12,000 \mathrm{~K}$ and $E(B-V)=0.86$ (continuous line). The spectra are normalized to the F814W flux and error bars corresponding to $0.1 \mathrm{mag}$ are shown. To all practical purposes the spectra are identical for wavelengths longer than $3300 \AA$, and only good data in the ultraviolet can help to distinguish them. Unfortunately, though, these data are often not available, as imaging in the ultraviolet can be done only from space and it requires very long exposure times for red stars. ${ }^{3}$ The purpose of the first step of the procedure, then, is to use a reddening-free color to eliminate the $E(B-V)-T_{\text {eff }}$ degeneracy when no ultraviolet observations are available.

Let us consider the reddening-free color $Q_{U B I}$, a combination of the magnitudes in the $U, B$, and $I$ passbands (e.g.,

\footnotetext{
${ }^{3}$ For example, $4700 \mathrm{~s}$ would be required to reach a signal-to-noise ratio of 5 in the F255W filter for an F0 V star at the distance of the Large Magellanic Cloud.
}

Mihalas \& Binney 1981, p186):

$$
Q_{U B I} \equiv(U-B)-\frac{E(U-B)}{E(B-I)}(B-I) .
$$

$Q_{U B I}$ is reddening-free in the sense that

$$
\begin{aligned}
Q_{U B I}= & (U-B)_{0}+E(U-B) \\
& -\frac{E(U-B)}{E(B-I)}\left[(B-I)_{0}+E(B-I)\right] \simeq Q_{U B I, 0},
\end{aligned}
$$

where the subscript " 0 " indicates the unreddened quantities. $Q_{U B I}$ is not exactly equal to $Q_{U B I, 0}$ because the coefficient in equation (2) is not a constant but depends on the temperature of the star and on the amount of reddening in front of it (see Appendix A). With the reddening law of Scuderi et al. (1996) and by using the WFPC2 filters F336W $(U)$, F439W $(B)$ and F814W $(I)$, one finds $E(\mathrm{~F} 336 \mathrm{~W}-\mathrm{F} 439 \mathrm{~W}) / E(\mathrm{~F} 439 \mathrm{~W}-\mathrm{F} 814 \mathrm{~W}) \simeq 0.42$ for $T_{\mathrm{eff}} \gtrsim$ 5500 , and this is the value we will use.

The reddening-free color $Q_{U B I}$ computed with the models by Bessel et al. (1998) for the WFPC2 filters is plotted in Figure 6 versus $\mathrm{F} 336 \mathrm{~W}-\mathrm{F} 814 \mathrm{~W}$. The curve is for $E(B-V)=0$, and the arrow is the reddening vector for $E(B-V)=0.2$ mag.

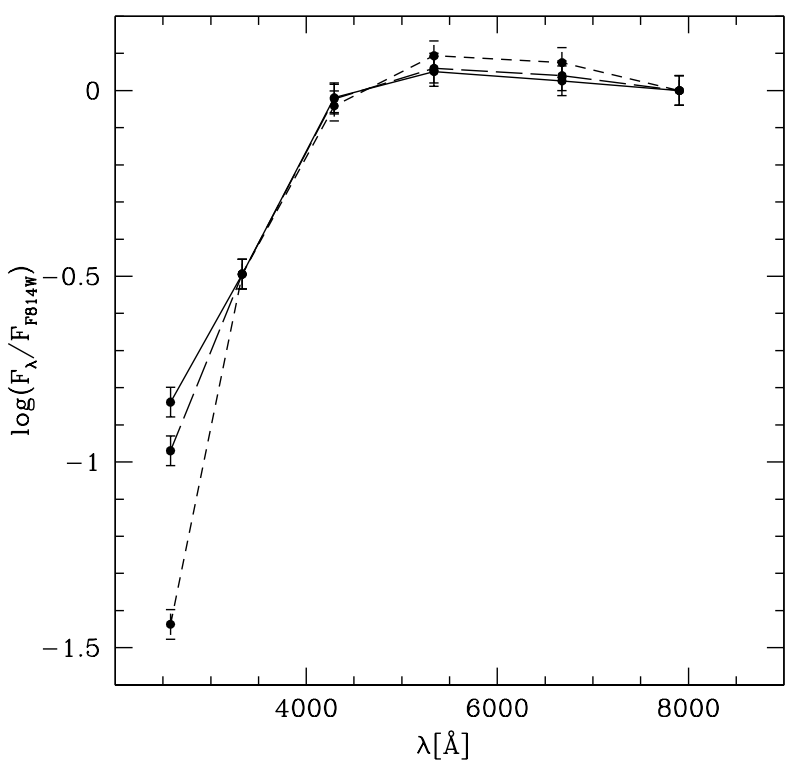

FIG. 5.-Comparison of spectra for different combinations of $T_{\text {eff }}$ and $E(B-V): T_{\text {eff }}=5000$ and $E(B-V)=0$ (short-dashed line), $T_{\text {eff }}=7600$ and $E(B-V)=0.55$ (long-dashed line), $T_{\mathrm{eff}}=12,000$ and $E(B-V)=0.86($ continuous line). The spectra are normalized to the F814W flux and error bars corresponding to $0.1 \mathrm{mag}$ are shown. As it can be seen, at this resolution the spectra are almost indistinguishable for wavelengths longer than $3300 \AA$, and ultraviolet imaging is needed to tell them apart. 


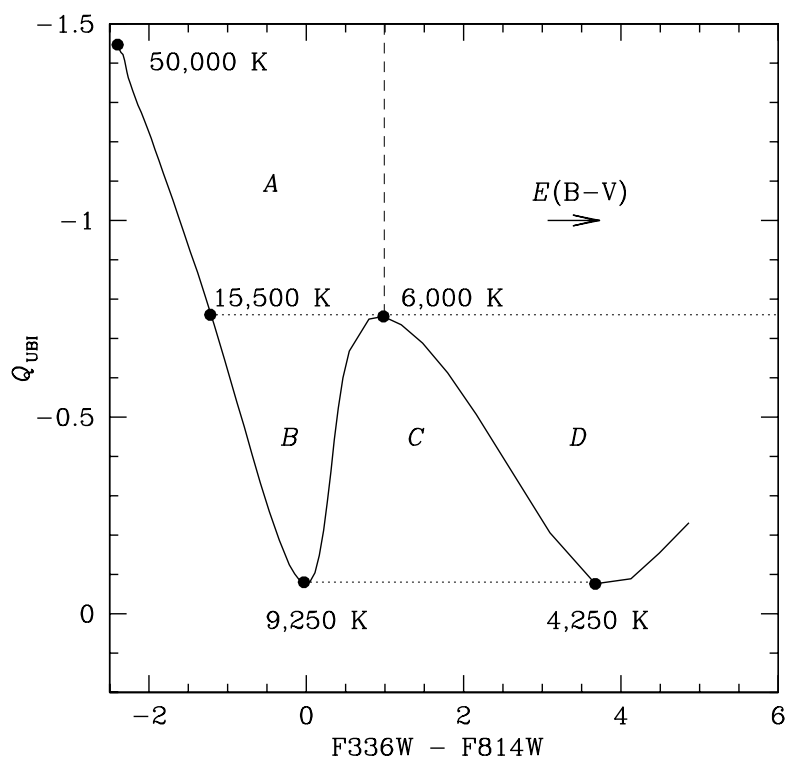

FIG. 6.-Reddening-free color $Q_{U B I}$ as a function of F336W-F814W from the theoretical models by Bessel, Castellani, \& Plez (1998) for $Z=0.3 Z_{\odot}$ and $\log g=4.5$. The horizontal arrow indicates the reddening vector for $E(B-V)=0.2 \mathrm{mag}$, and a few important temperatures along the sequence are also marked. Stars in the four regions A-D have a different number of solutions (see text). The dashed line highlights the location of the T Tauri stars: their colors are not well represented by normal photospheres.

Since, by construction, the value of $Q_{U B I}$ does not change with $E(B-V)$, the effect of reddening in the $Q_{U B I}$ versus $\mathrm{F} 336 \mathrm{~W}-\mathrm{F} 814 \mathrm{~W}$ plane is to move the points horizontally to the right from the zero-reddening locus, as indicated by the arrow in Figure 6. Moreover, $\Delta(\mathrm{F} 336 \mathrm{~W}-\mathrm{F} 814 \mathrm{~W})$, the horizontal distance of an observed point from the theoretical zero-reddening curve, is proportional to $E(B-V)$ :

$$
\begin{aligned}
\Delta(\mathrm{F} 336 \mathrm{~W}-\mathrm{F} 814 \mathrm{~W})= & E(\mathrm{~F} 336 \mathrm{~W}-\mathrm{F} 814 \mathrm{~W}) \\
& \simeq 3.3 E(B-V),
\end{aligned}
$$

whereas the observed $Q_{U B I}$ is, to first approximation, a function of only the star's temperature (see Fig. 6).

As is apparent from Figure 6, $Q_{U B I}$ is not a monotonic function of $\mathrm{F} 336 \mathrm{~W}-\mathrm{F} 814 \mathrm{~W}$, i.e., temperature, but it is W-shaped. ${ }^{4}$ This, in turn, is due to the nonmonotonicity of the Balmer jump, the intensity of which peaks for A0 stars and declines both for earlier and later spectral types (see, for example, Allen 1973, p207). So, stars in region $A$ on Figure 6 have only one possible intersection with the zero-reddening curve and for them the solution $T_{\text {eff }}-E(B-V)$ is unique. In the case of stars in region $B$, there are three intersections with the reddening curve, but the two on the right are easily rejected by noticing that, according to equation (3), they correspond to negative values of $E(B-V)$ and, hence, are not acceptable. The solution, therefore, even if not unique, is unambiguous.

Things are more complicated for the stars with F336W-F814W $\gtrsim 0$, i.e., $T_{\text {eff }} \lesssim 9000 \mathrm{~K}$. Here, the solution is not only nonunique, but also ambiguous. As a matter of fact, there are two (region C) or three (region D) combinations of $T_{\text {eff }}$ and $E(B-V)$ that are absolutely equivalent. All

${ }^{4}$ The additional hook at $\mathrm{F} 336 \mathrm{~W}-\mathrm{F} 814 \mathrm{~W} \gtrsim 3.5$ is caused by the WFPC22 F336W filter red leak. these solutions are physical and there is no a priori criterion to decide which one is the right one.

The first step of the procedure is to divide the stars in four classes, according to their photometric error and the number of solutions in the $T_{\text {eff }}-E(B-V)$ plane. In other words, they are classified according to the mean error in five bands, $\bar{\delta}_{5}$, introduced in equation (1) and to the number of intersections that they have to their left, i.e., bluer F336W-F814W color, with the theoretical zero-reddening curve in the $Q_{U B I}$ versus $\mathrm{F} 336 \mathrm{~W}-\mathrm{F} 814 \mathrm{~W}$ plane. To account for the photometric errors, we project the measured value plus or minus its error. In the following, a star belongs to a certain class if the measured point and the $\pm 2 \sigma$ values have the same number of intersections.

The classes are defined as follows:

Class I: Stars that have only one intersection to their left. They are the stars in region $A$ and $B$ in Figure 6.

Class II: Stars in region $C$ in Figure 6. They have two intersections to their left. The solution for $E(B-V)$ and $T_{\text {eff }}$ is not unique, and we shall see shortly how we choose between the different possibilities.

Class III: Stars that, in spite of their good photometry $\left(\bar{\delta}_{5}<0.1\right)$, have a "problematic" location in the $Q_{U B I}$ versus F336W-F814W plane. These are

a) Stars whose horizontal projection never intersects the zero-reddening curve to their left.

b) Stars that belong to region $D$ Figure 6 and, hence, have three intersections to their left. As we shall discuss in $\S 6$, in this region the particular combination of colors we have chosen is sensitive to metallicity, and this classification scheme is not reliable.

c) Stars that fall above the peak at $\mathrm{F} 336 \mathrm{~W}-\mathrm{F} 814 \mathrm{~W}=1$, roughly along the long-dashed line in Figure 6, for which the F336W flux is too strong compared with that in the other bands. As discussed in Panagia et al. (2000), they are UV excess objects, most likely T Tauri stars, and the $U$-band excess is due to the presence of a circumstellar disk accreting onto the star. As a consequence, their spectrum is not well modeled by the one of a normal photosphere, and no reliable $E(B-V)$ can be derived by comparing their colors with those of a normal photosphere.

Class IV: Finally, stars with poor overall photometry, i.e., $\bar{\delta}_{5}>0.1$, are assigned to this class, independent of the number of intersections.

As an example, the result of the division into classes is shown in Figure 7 for the WF3 chip of the 1997 July observations. Class I stars are hotter than about 10,000 K, whereas class II stars are between 6750 and $8500 \mathrm{~K}$. In the particular case we are discussing here, out of a total number of 3139 stars, 40 belong to class I (1.3\%), 388 to class II $(12.4 \%)$, and 615 to class III (19.6\%). The rest of them (2096 or $66.8 \%$ ) belong to class IV.

Again, for every star the measured $Q_{U B I}$ gives a first-order estimate of the temperature (see Fig. 6), and the excess in $E(\mathrm{~F} 336 \mathrm{~W}-\mathrm{F} 814 \mathrm{~W})$ gives a first-order estimate of $E(B-V)$ from equation (3). These are, then, used as an input in the second step of the procedure, the six-band fit, which we will now describe.

\subsection{Second Step: Six-Band Fit}

In the first step we have used only a subset of the six bands available. Now that the number of possible solutions in the 


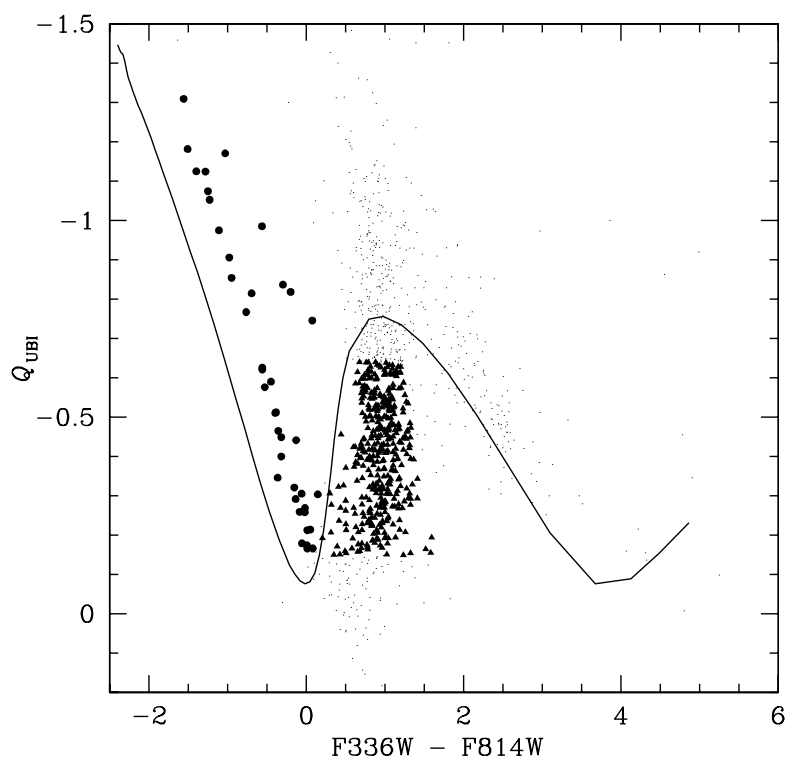

FIG. 7.--Location of class I (filled circles), II (filled triangles), and III (small dots) stars in the $Q_{U B I}$ vs. F336W-F814W plane.

$T_{\text {eff }}-E(B-V)$ plane is assessed for every star, we can proceed to fit the entire spectrum at once with a $\chi^{2}$ technique using the information gathered in the first step as a starting point for the fit:

Class I: The fit is performed in a neighborhood of \pm 0.1 mag around the $E(B-V)$ value deducted from the projection in the $Q_{U B I}$ versus $\mathrm{F} 336 \mathrm{~W}-\mathrm{F} 814 \mathrm{~W}$ plane.

Class II: We choose the smallest of the two $E(B-V)$ values from the projection described above as the starting point and perform the fit in a neighborhood around it, defined to avoid the other possible solutions. This is somewhat arbitrary, but an inspection of Figure 7, for example, does not indicate any compelling evidence for a large number of stars with extinction much larger than the mean value. Let us stress here that the reddening is still computed individually for each star.

Classes III and IV: For the various reasons mentioned above, photometry is not reliable enough to allow us solve for all three unknowns simultaneously, and the reddening is derived from class I and II stars. We have explored two options: either using the mean value of the four closest class I and II neighbors or the mean over the entire field. In the case of the field of SN 1987A the result is the same, as the extinction does not show any significant spatial pattern and the local mean is mostly equal to the general one. However, when the reddening shows appreciable small-scale correlations, the local mean does give better results, most notably a narrower main sequence, and the first option should be preferred (see, for example, the case of NGC 6822 in Bianchi et al. 2001).

The details of the $\chi^{2}$ fit are described in Appendix B. While in the selection process we have used constant extinction coefficients to build $Q_{U B I}$ (see eq. [2]), in the six-band fit they vary according to the $T_{\text {eff }}$ and $E(B-V)$ values (see Table 8 in Appendix A).

This procedure assures total control over the fitting process. In fact, even in the case of multiple intersections, it provides a criterion to choose the most reasonable solution. Once this is done, the six-band fit uses all the information available and thus gives the best possible answer. Moreover,

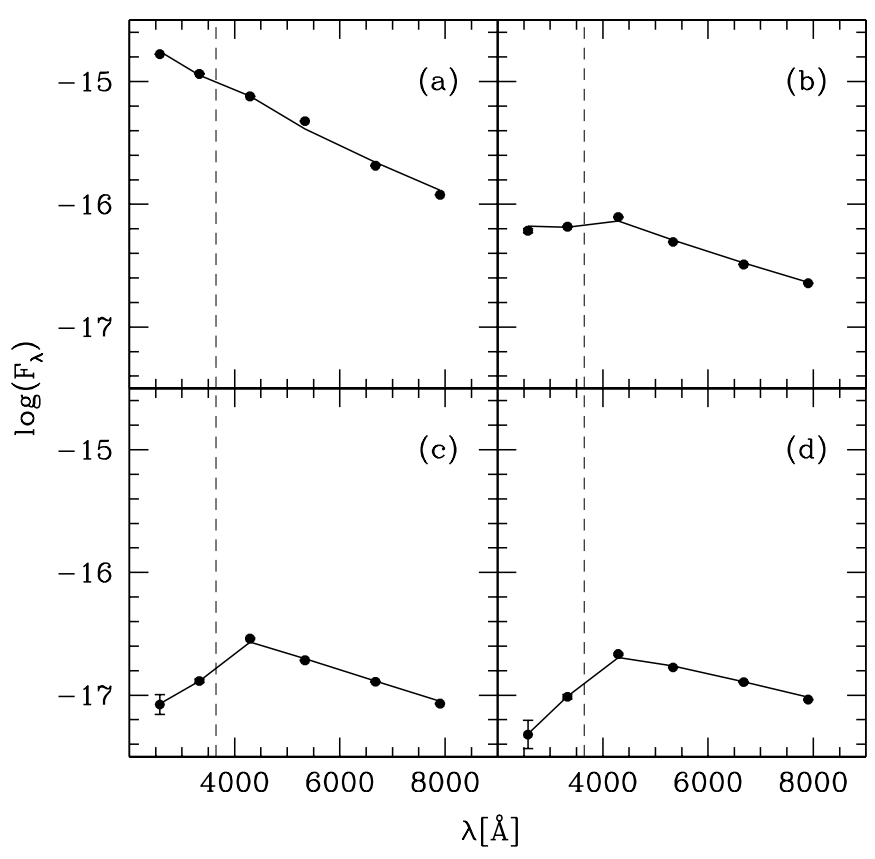

FIG. 8.- Same as Fig. 4, but showing the best-fitting model as a continuous line. The parameters derived from the fit are listed in Table 4.

the whole procedure is very efficient in terms of computer CPU time usage, since it limits the size of the grid in the sixband fit, which is the most time-consuming part of it all, to only a small region in the parameter space around the physical minimum in the $T_{\text {eff }} E(B-V)$ plane.

\section{RESULTS OF THE FIT}

As an example of the results of the procedure, in Figure 8 we show the spectra of the same four stars of Figure 4 together with the best-fitting models. The derived parameters and their errors are listed in Table 4.

The H-R diagram for the 21,955 stars in the field of SN 1987A is displayed in Figure 9. It is interesting to compare it to what one would have obtained from a "blind" fit of the spectrum, i.e., by skipping the selection described in $\S 4.1$ and performing directly the six-band fit of $\S 4.2$. This is shown in Figure 10 (left).

The fit for the stars marked in gray in Figure 10 (left) is obviously wrong, as they occupy an impossible region of the $\mathrm{H}-\mathrm{R}$ diagram. An inspection of Figure 10 (right) reveals that all these stars, and only they, were fitted with too high a value of $E(B-V)$ and, consequently, of $T_{\text {eff. These are all }}$

TABLE 4

Best-Fit Parameters for the Spectra of Figure 8

\begin{tabular}{ccccc}
\hline \hline \multirow{2}{*}{$\begin{array}{c}T_{\text {eff }} \\
\text { Panel }\end{array}$} & $(\mathrm{K})$ & $E(B-V)$ & $\log \left(L / L_{\odot}\right)$ & $R / R_{\odot}$ \\
\hline$a \ldots \ldots \ldots$. & $25,350 \pm 5,700$ & $0.202 \pm 0.080$ & $3.662 \pm 0.264$ & $3.444 \pm 0.090$ \\
$b \ldots \ldots \ldots .$. & $18,160 \pm 2,100$ & $0.431 \pm 0.051$ & $2.695 \pm 0.166$ & $2.265 \pm 0.050$ \\
$c \ldots \ldots \ldots .$. & $8,000 \pm 350$ & $0.117 \pm 0.050$ & $1.176 \pm 0.079$ & $2.070 \pm 0.042$ \\
d......... & $7,480 \pm 300$ & $0.209 \pm 0.047$ & $1.255 \pm 0.067$ & $2.548 \pm 0.052$ \\
\hline
\end{tabular}

Note.-The star in Fig. $8 a$ is saturated, and this causes the large quoted errors on the quantities derived for it. The corresponding magnitudes are listed in Table 3. 


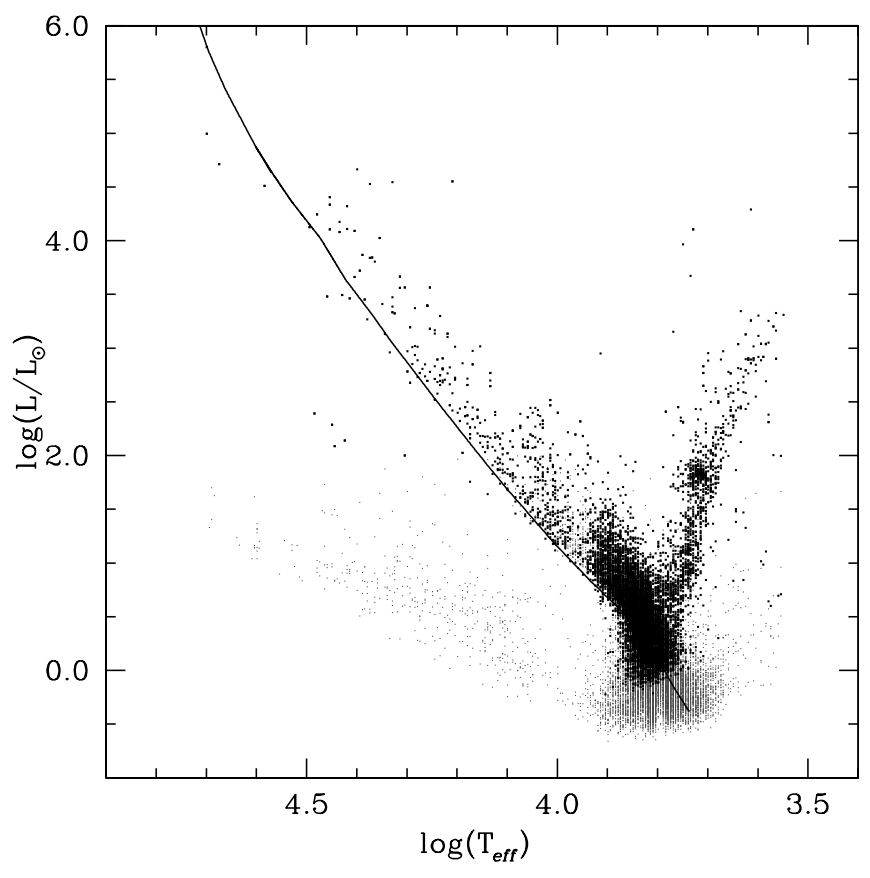

FIG. 9.- H-R diagram for the stars in the field around SN 1987A, showing the 9474 stars with $\delta \log \left(T_{\text {eff }}\right)<0.05$ (black dots) and the zero-age main sequence for $Z=0.3 Z$ from the models by Brocato \& Castellani (1993) and Cassisi, Castellani, \& Straniero (1994) for masses below $25 M_{\odot}$ and by Schaerer et al. (1993) for those above $25 M_{\odot}$ (continuous line).

stars that fall in region $C$ or $D$ in Figure 6 for which the broadband spectrum can be interpreted either as a heavily reddened hot star or as a cold one with little dust in front of it. The fit just selects the solution with the lowest $\chi^{2}$, even if it is marginally lower than other possible minima. Which one of the possible solutions has the lowest $\chi^{2}$ depends on such things as the $E(B-V)$ and $T_{\text {eff }}$ grids used in the fit and, in the case shown in Figure 10, the wrong one is chosen for 2 stars out of 3. For many of these stars the available photometry is limited to F439W and redder filters. As we will see in $\S 7$, then, the data are very little sensitive to temperature, and these cold stars can be mistaken for objects as hot as $50,000 \mathrm{~K}$.

On the other hand, selecting the right solution before performing the fit eliminates the problem almost completely. There still are roughly 600 stars (less than $3 \%$ of the total) under the zero-age main-sequence locus. However, they are mostly class IV objects, i.e., stars with very poor photometry, or stars with peculiar colors, i.e., the T Tauri stars described in detail by Panagia et al. (2000), or unresolved binaries.

The stars with multiple solutions are the late-type ones. Being able to measure directly the reddening for them is important for two reasons. First, as low-mass stars are much more numerous than higher mass ones, the number of reddening determinations increases dramatically. In fact the late-type stars for which eventually we are able to measure the reddening are typically a factor of 10 more numerous than those earlier than A0. Second, stars of different masses have different lifetimes and there is no a priori reason why different generations of stars should be affected by the same amount of extinction (see Zaritsky 1999, for an example in the LMC itself). By extending our analysis to a wide range of masses we can check for population-dependent effects and deredden the different generations of stars in the most appropriate way.

In addition to measuring the intrinsic properties of the stars shown in Figure 9, our procedure also provides individual $E(B-V)$ measurements for more than 2500 stars, on average 1 every $13 \operatorname{arcsec}^{2}$. The resulting histogram and spa-
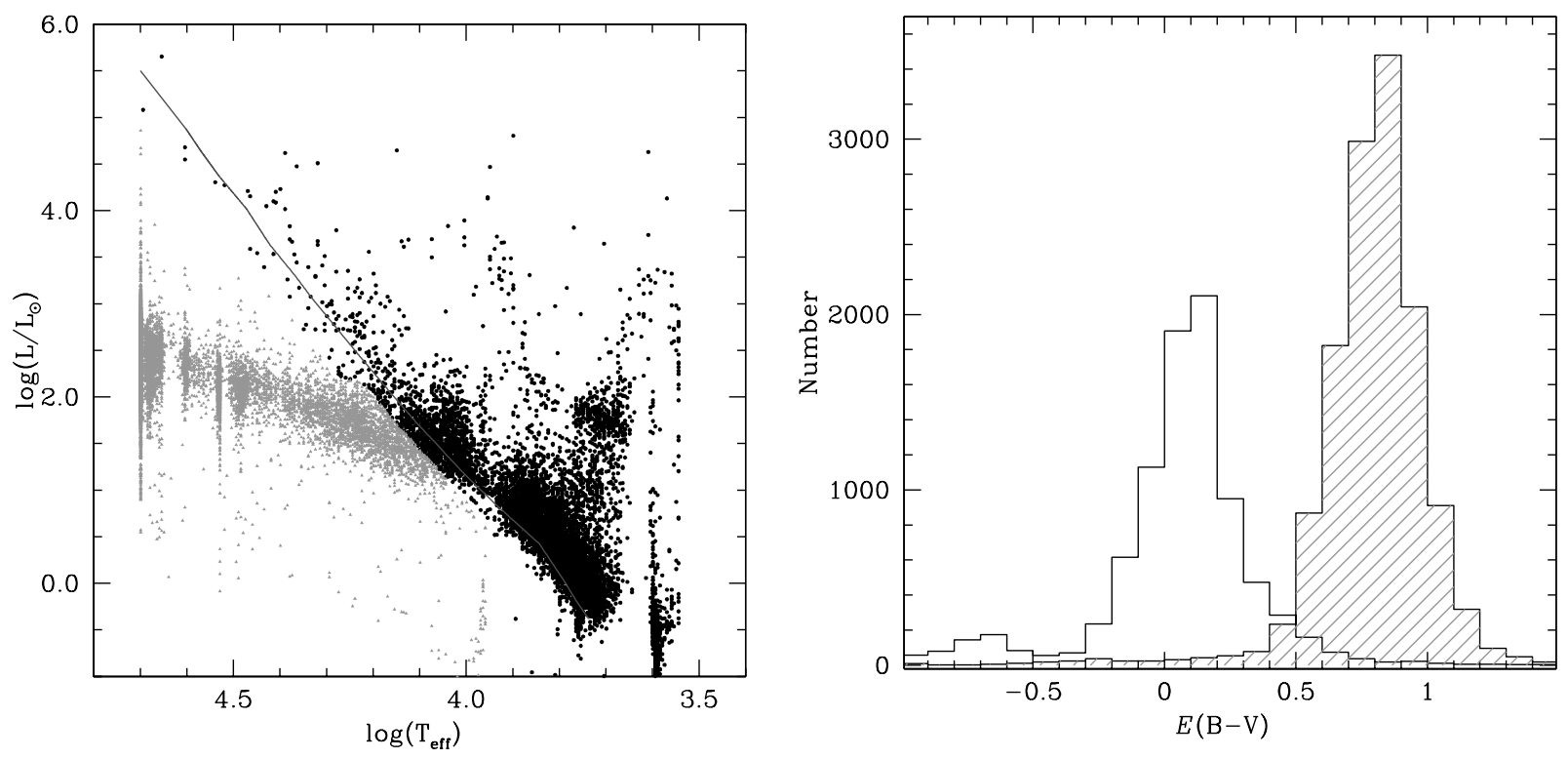

FIG. 10.- Results of the "blind" fit, for the stars around SN 1987A: the fit described in $\S 4.2$ is performed directly without the selection of $\S$ 4.1. Left: H-R diagram. The gray triangles highlight the stars for which the fit returns too high a value of $T_{\text {eff }}$ and $E(B-V)$. These are stars with multiple solutions for which the "blind" fit picks the wrong one (see text). The zero-age main sequence for $Z=0.3 Z_{\odot}$ from the models by Brocato \& Castellani (1993) and Cassisi et al. (1994) for masses below $25 M_{\odot}$ and by Schaerer et al. (1993) for those above $25 M_{\odot}$ is shown as a continuous line. Right: $E(B-V)$ distribution resulting from the "blind" fit. The high reddening peak (hatched histogram) is entirely made of stars plotted in gray in the left panel, thus confirming that their location in the $\mathrm{H}-\mathrm{R}$ diagram is due to an erroneous reddening determination. 
tial distribution of interstellar reddening are shown in Figure 11 (see also Panagia et al. 2000). As it can be seen, the initial suspicion is confirmed and the reddening is indeed very patchy and varies on scales of a few arcseconds.

Besides providing a very accurate reddening map, measuring individual values of $E(B-V)$ also leads to a narrowing of the various features in the color-magnitude diagram, in particular the main sequence. This is of great help when interpreting the data, because it allows one to disentangle the broadening due to differential extinction from the one caused, for example, by the overlap of several generations of stars or by the presence of binary stars.

In Figure 12 we compare the stars in the F439W versus (F439W-F814) diagram before and after reddening correction. The density contours in $(a)$ and $(b)$ are spaced by factors of 2. Apart from the obvious fact that the corrected stars are bluer and more luminous, it is apparent that the features in the reddening-corrected diagram are narrower than the corresponding ones in the observed CMD. To quantify this effect let us consider a color cut through the main sequence, such as the one displayed in Figure $12 c$ (see also Fig. 13). Here, the blue line histogram represents the color distribution of the dereddened main-sequence stars with $\bar{\delta}_{5}<0.1$ in the $21 \mathrm{mag}>m(\mathrm{~F} 439 \mathrm{~W})_{0}>22$ mag interval. The red line histogram gives the distribution of the same stars before reddening correction. To facilitate the comparison, this latter is shifted in color so as to have the same mode as the dereddened one. As is apparent, the original distribution is definitely more spread out in color: its rms is $24 \%$ larger than the corrected one ( 0.21 versus $0.17 \mathrm{mag})$. This is the case all along the main sequence, as shown in Figure 13, where we plot the color distribution for all the stars with $\bar{\delta}_{5}<0.1$ in different magnitude bins. The dereddened histograms (blue line) always display a smaller scatter than the observed ones (red line), confirming the effectiveness of the correction procedure.

As it can be expected, the color distribution is even narrower when only the stars with individual reddening deter- minations, i.e., those we have classified as class I and II, are considered. This is shown in Figure 14. In this case the rms of the reddening-corrected main-sequence histogram, again in the $21 \mathrm{mag}>m(\mathrm{~F} 439 \mathrm{~W})_{0}>22 \mathrm{mag}$ interval, is 0.14 mag, while the one of the same stars before correction is $0.18 \mathrm{mag}$, i.e., 29\% larger. A simple Kolmogorov-Smirnov test shows that the widths of the two distributions are different at the $99.9995 \%$ level.

To conclude, let us remark again that class III and IV stars were not corrected with an individually determined value of $E(B-V)$, but, rather, with the mean value from 2510 class I and II neighbors. It is important to realize that, given the large number of these stars, the mean reddening used to correct class III and IV stars is on average very accurate. Of course, it is still possible that a few class III and IV stars have, in reality, extinction values significantly different from their local mean, but the global effect is negligible.

The full discussion of the stellar content of the region around SN 1987A can be found in Romaniello (1998) and Panagia et al. (2000).

\section{ROLE OF GRAVITY AND METALLICITY}

So far we have used model atmospheres with only one surface gravity ( $\log g=4.5$, appropriate for main-sequence stars $)$ and metallicity $\left(Z=0.3 Z_{\odot}\right.$, the expected mean value for the LMC). Even though these are sensible assumptions for the environment under study, we have to verify their influence on the results we derive.

Let us start by considering the surface gravity. The theoretical zero-reddening locus in the $Q_{U B I}$ versus F336W-F814W plane is shown in Figure 15 for three values of surface gravity, namely, $\log g=4$ (dotted line), 4.5 (continuous line), and 5 (dashed line). As it can be seen, the three curves overlap perfectly for F336W-F814W $\lesssim-0.5$, i.e., $T_{\text {eff }} \gtrsim 11,000 \mathrm{~K}$, and $\mathrm{F} 336 \mathrm{~W}-\mathrm{F} 814 \mathrm{~W} \gtrsim 1.5$, i.e., $T_{\text {eff }} \lesssim 5500 \mathrm{~K}$. Elsewhere, however, different surface gravities lead to different expected zero-reddening loci in the
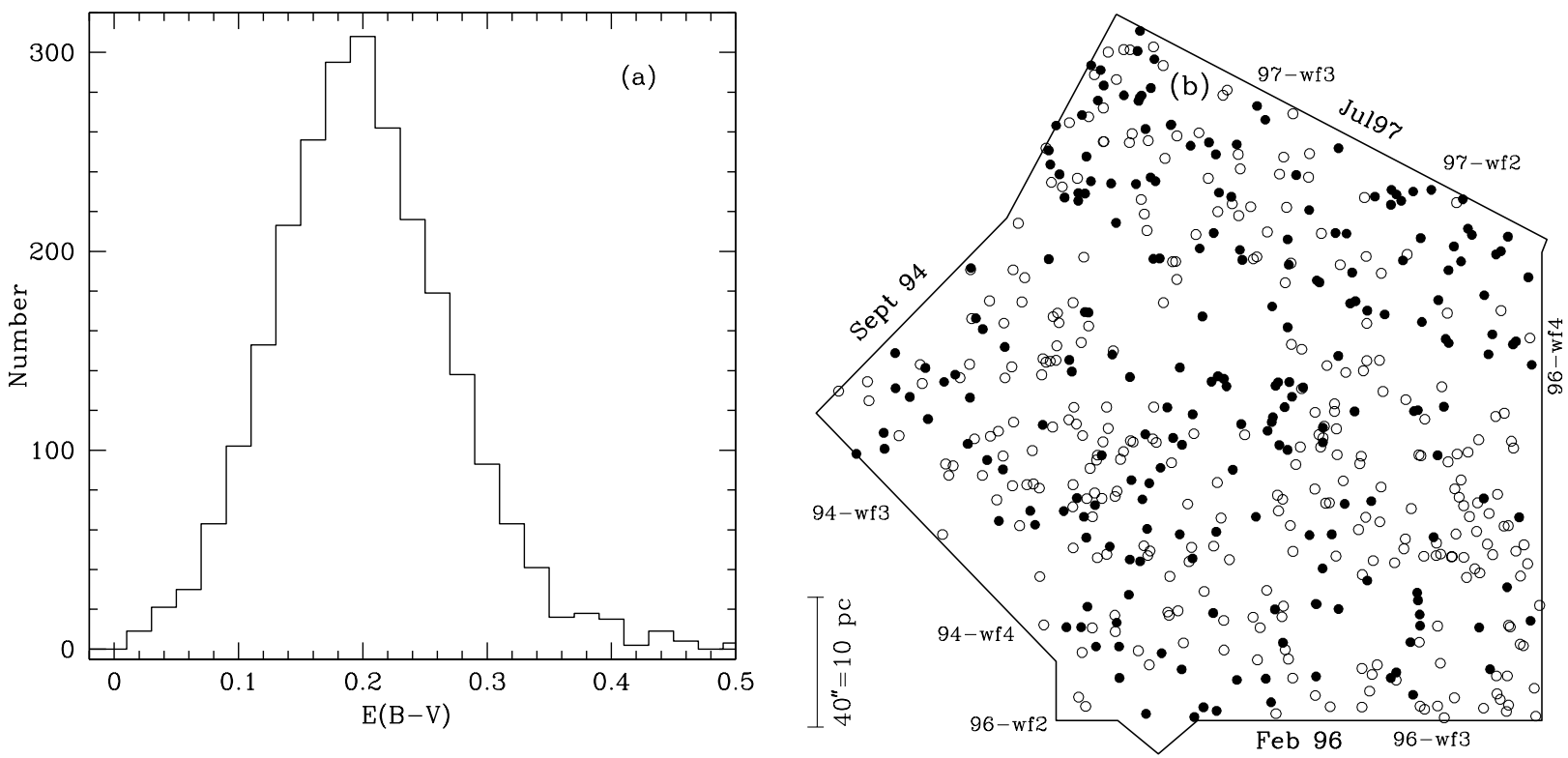

FIG. 11. - (a) Reddening histogram from the stars in the field of SN 1987A. Only stars for which $E(B-V)$ was measured individually (the 2510 class I and II stars; see the text) are used here. (b) Spatial distribution of stars with high reddening $[E(B-V)>0.3]$ ( filled circles) and low reddening $[E(B-V)<0.1]($ open circles). 

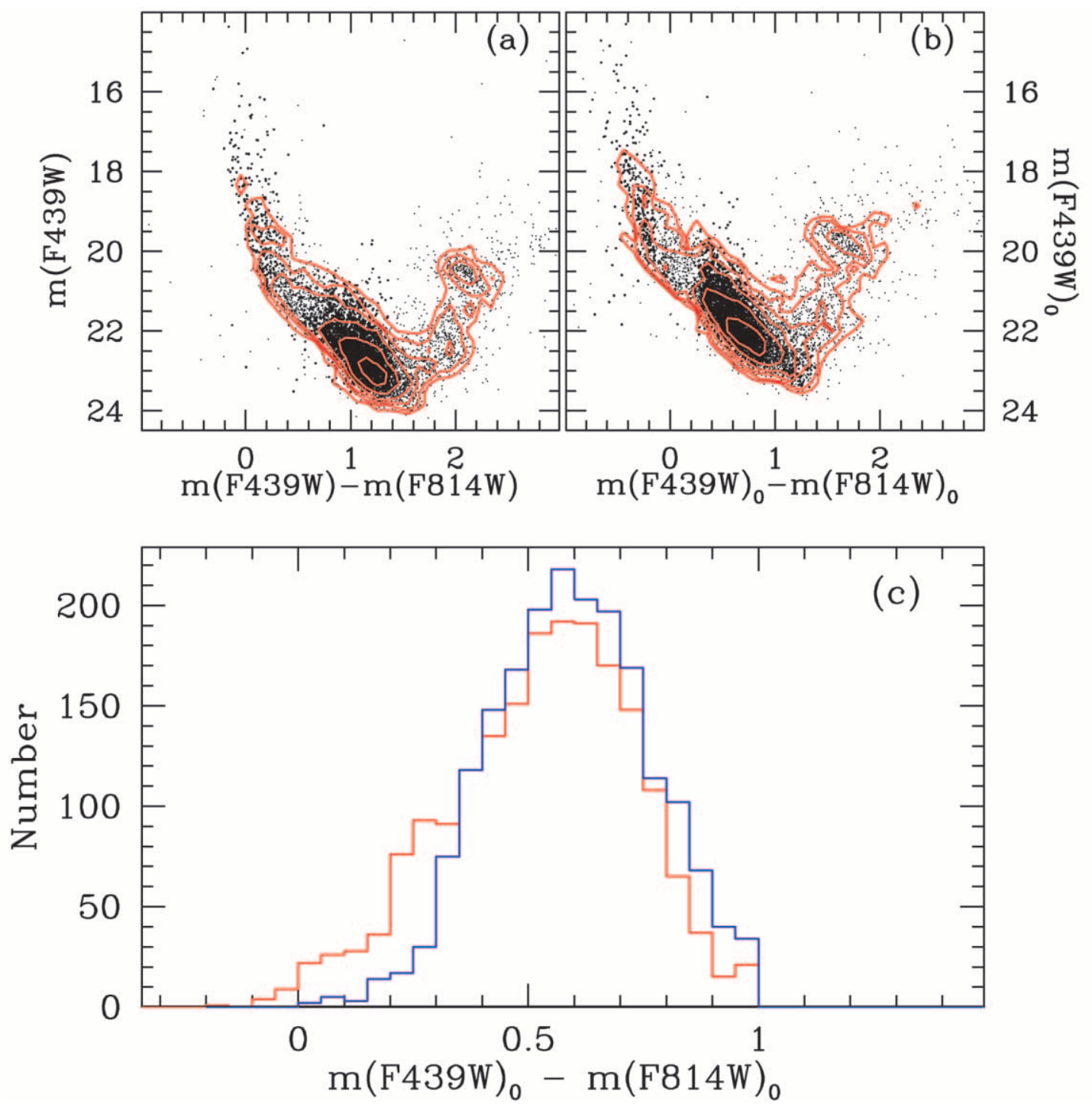

FIG. 12.-Before and after the reddening correction. (a) Observed F439W vs. F439W-F814W diagram for all the stars in our WFPC2 frames with $\bar{\delta}_{5}<0.1$; (b) same as $(a)$, but dereddened. Black dots represent the stars for which $E(B-V)$ was determined individually (class I and II; see text); gray dots, stars dereddened with the mean value of $E(B-V)$ from the closest class I and II neighbors. The density contours, spaced by factors of 2, are superposed in red. The visual impression that the main sequence in $(b)$ is narrower than the one in $(a)$ is confirmed by the histograms in $(c)$. Here, the blue line represents the color distribution of the dereddened stars in the $21 \mathrm{mag}<m(\mathrm{~F} 439 \mathrm{~W})_{0}<22 \mathrm{mag}$ interval, and the red one, the distribution of the observed colors for the same stars. For the sake of clarity, the latter was shifted by $0.5 \mathrm{mag}$ in color to have the same mode as the dereddened one. There are 218 and 192 stars at the peak of the dereddened and observed distribution, respectively, while each histogram contains a total of 1923 stars.

$Q_{U B I}$ versus $\mathrm{F} 336 \mathrm{~W}-\mathrm{F} 814 \mathrm{~W}$ diagram. In particular, this influences the division in classes that is the foundation of the method described above.

However, as it can be seen, for example, in Figure 14, only stars on or near the main sequence are used to determine the reddening. Adopting the stellar evolutionary models by Brocato \& Castellani (1993) and Cassisi, Castellani, \& Straniero (1994), we see that for $Z=0.3 Z_{\odot}$ the gravity is nearly constant along the main sequence and that its value is $\log g=4.5$, the value we have used in the fit. This is a sound assumption over a large range of metallicities because extensive model calculations show that surface gravity variations as a function of chemical composition are quite small. For example, at $(\mathrm{F} 336 \mathrm{~W}-\mathrm{F} 814 \mathrm{~W})_{0}=0$ the surface gravity ranges from $\log g \simeq 4.4$ for a solar-metallicity star to $\log g \simeq 4.6$ for $Z=Z_{\odot} / 20$. Considering that for every metallicity the gravity is almost constant along the main sequence one can safely adopt the value of $\log g=4.5$, independent of chemical composition.

Once a star evolves off the main sequence its surface gravity changes appreciably. For example, $\log g \simeq 3$ is a typical value for the stars in the red giant clump, i.e., the stars at $\log \left(T_{\text {eff }}\right) \simeq 3.7$ and $\log \left(L / L_{\odot}\right) \simeq 1.75$ in Figure 9. Even though the $Q_{U B I}$ versus F $336 \mathrm{~W}-\mathrm{F} 814 \mathrm{~W}$ relation is, indeed, sensitive to gravity, the result of our global fit is not. This is because, as we have seen, we do not fit simultaneously temperature and reddening for stars off the main sequence, but rather we assign to them the mean $E(B-V)$ value of their class I and II neighbors, which are on the main sequence and hence have $\log g \simeq 4.5$. For stars that are off the main sequence, we just perform the fit to solve for the radius and effective temperature. Once the reddening is correctly determined, the result of the multiband fit is no longer sensitive to the surface gravity adopted in computing the theoretical 


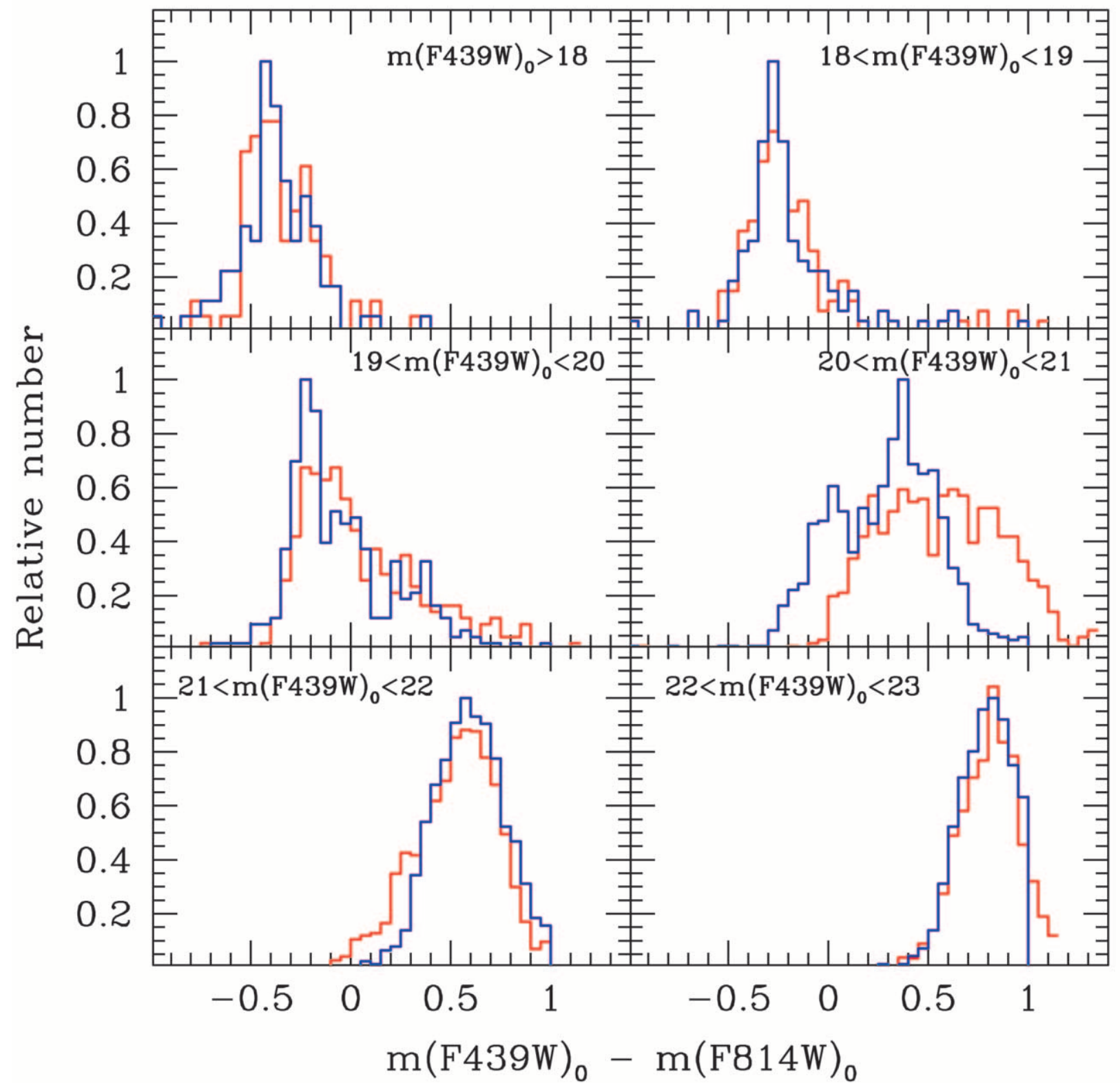

FIG. 13. - Width of the main sequence as a function of magnitude before (red line) and after (blue line) reddening correction. The magnitude interval along the main sequence is indicated in each panel. The faintest magnitude bin suffers heavily from incompleteness. Also, the raw histograms were shifted in color to have the same mode as the corrected ones. As can be seen, the corrected histograms are systematically narrower than the uncorrected ones.

colors. We have checked this by dividing the stars into classes by using the $Q_{U B I}$ versus F336W-F814W relation for $\log g=4.5$, and then fitting the stars with colors computed for various values of the gravity. The maximum variations in temperature for the stars on the red giant branch are of $3 \%$, i.e., much smaller than the error deriving from the fit itself, with no systematic trend with gravity. Given this result, we have decided to use $\log g=4.5$ all throughout our analysis.

In addition to influencing the stellar structure, the chemical composition also plays a role in determining the star's spectrum, through the atmospheric opacity. As a consequence, the colors also depend on this parameter. The $Q_{U B I}$ versus $\mathrm{F} 336 \mathrm{~W}-\mathrm{F} 814 \mathrm{~W}$ relation for different values of $Z$ is shown in Figure 16. Again, as it can be seen, the variations are negligible for the stars for which the reddening is an individual fit parameter, i.e., class I and II stars.

Also in this case, then, once the division into classes is made and the local mean reddening for class III and IV stars is determined from the individual values of class I and II neighbors, the result of the multiband fit is insensitive to the details of the stellar atmospheres. The comparison of the fits performed with colors computed for different metallicities shows that the derived parameters do not depend appreciably on metallicity.

We conclude that in applying our fitting procedure, as described in $\S 4$, one can safely use colors computed with models by Bessel et al. (1998) for $Z=0.3 Z_{\odot}$ and $\log g=4.5$ to derive accurate stellar parameters and interstellar absorptions for all stars. 

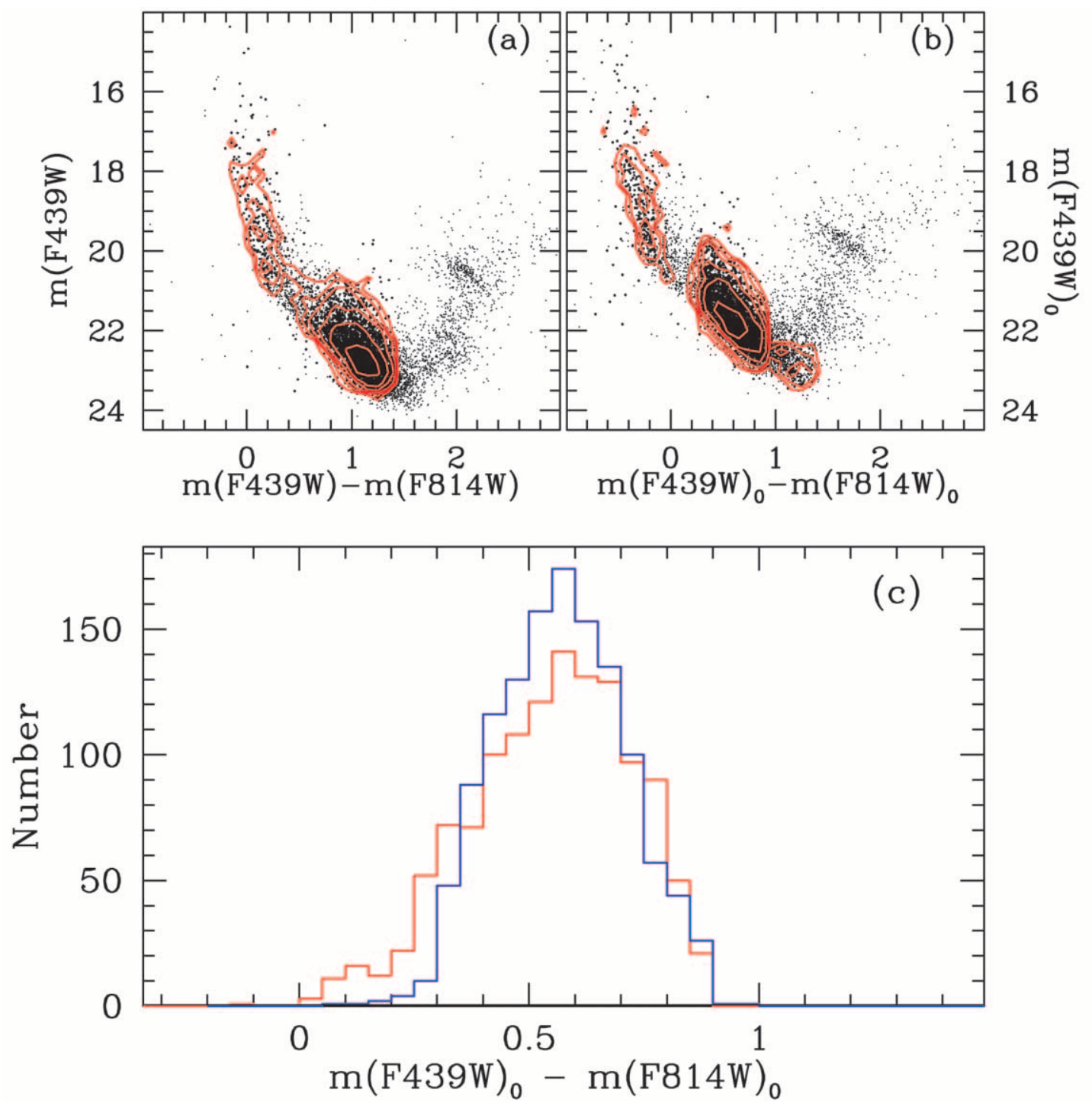

FIG. 14. - Same as Fig. 12, but the contours in $(a)$ and $(b)$ and the histograms in $(c)$ refer only to the stars with individual reddening correction (class I and II; see text). The peak values of the histograms in (c) are 174 and 141 for the corrected (blue line) and observed one (red line), respectively.

\section{BANDS NEEDED}

For every star there are three unknown quantities: the angular radius, the effective temperature, and the reddening. Therefore, for each star one needs at least data in three bands to make the problem mathematically well defined. For good results the measurements in those three bands must also be rather accurate, thus requiring that the bands be selected to fall around the wavelength of the maximum of the spectral distribution of any given star and to have a suitable wavelength spacing to assure a sufficient baseline for the spectral shape analysis. In practice this is achieved with bands separated from each other by about $30 \%-40 \%$ of their central wavelengths. Moreover, when dealing with a stellar population one wants to study stars within a wide range of temperatures, and, therefore, more than three bands are absolutely needed. In this section we will investigate how many and which bands are needed to constrain the fit.

Let us start by noting that a $U$-like band is mandatory to recover both $T_{\text {eff }}$ and $E(B-V)$. To illustrate this point let us define the reddening-free color $Q_{U B I}$ combination of $B, V$, and $I$. By analogy with equation (2),

$$
Q_{B V I} \equiv(B-V)-\frac{E(B-V)}{E(V-I)}(V-I) .
$$

$Q_{B V I}$ is shown in Figure 17 as a function of F $439 \mathrm{~W}-\mathrm{F} 814 \mathrm{~W}$ (left) and $T_{\text {eff }}(r i g h t)$.

Since the $B, V$, and $I$ filters do not include the Balmer jump, the relation between $Q_{B V I}$ and $B-I$ is monotonic, ${ }^{5}$ thus solving the problem of ambiguities in the solution. However, one immediately notices that the slope of the curve is much shallower than in the $Q_{U B I}$ case. In fact, the total excursion of $Q_{B V I}$ between 3500 and $50,000 \mathrm{~K}$ is only $0.4 \mathrm{mag}$, and it is almost totally insensitive to temperatures higher than $6000 \mathrm{~K}$, i.e., F439W-F814W $\lesssim 1$.

The combined effect of the weak dependence of $Q_{B V I}$ on temperature and photometric errors is to make every combination $E(B-V)-T_{\text {eff }}$ along the reddening vector essen-

${ }^{5}$ The hook at very low temperatures is due to the red leak in the WFPC2 F439W filter. 


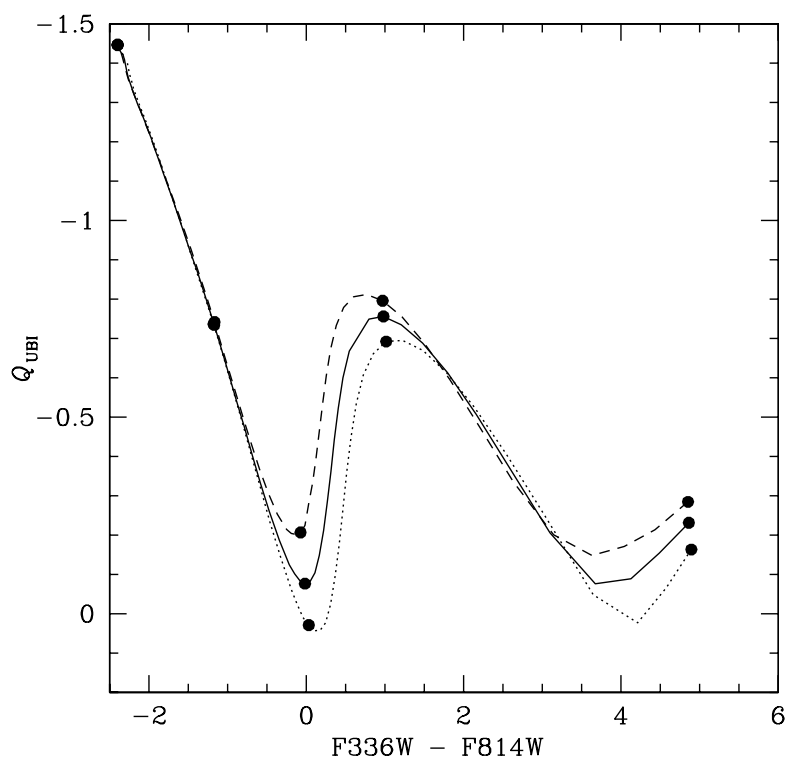

FIG. 15.-Zero-reddening $Q_{U B I}$ vs. F336W-F814W relation for three values of surface gravity: $\log g=5$ (dashed line), $\log g=4.5$ (continuous line), and $\log g=4$ (dotted line). The model atmospheres are those of Bessel et al. (1998) for $Z=0.3 Z_{\odot}$. The points for $T_{\text {eff }}=50,000,15,000,9250$, 6000 , and $3500 \mathrm{~K}$ are marked on each curve.

tially equivalent. This is shown in Figure 18 and Figure 19, where $\chi^{2}$ maps of the fit using only four bands $(\mathrm{F} 439 \mathrm{~W}$, F555W, F675W, and F814W; top) are compared with those using all six of them (bottom). As it can be seen, when only four bands are used the $\chi^{2}$ has no distinct minima, and, hence, it is not possible to determine both the reddening and the temperature, either for hot stars for which the solution is unambiguous (Fig. 18) nor for the cold ones with multiple minima (Fig. 19).

Also, let us note here that, while observations at wavelengths shorter than the Balmer jump are mandatory to recover both $T_{\text {eff }}$ and $E(B-V)$, it is preferable not to go too far in the ultraviolet, unless one can afford to make observa-

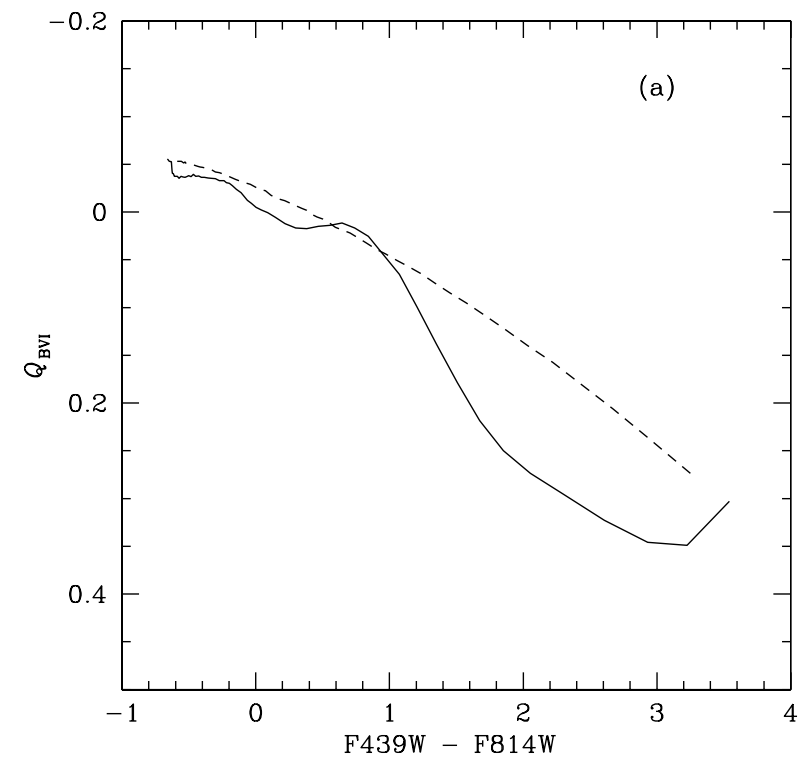

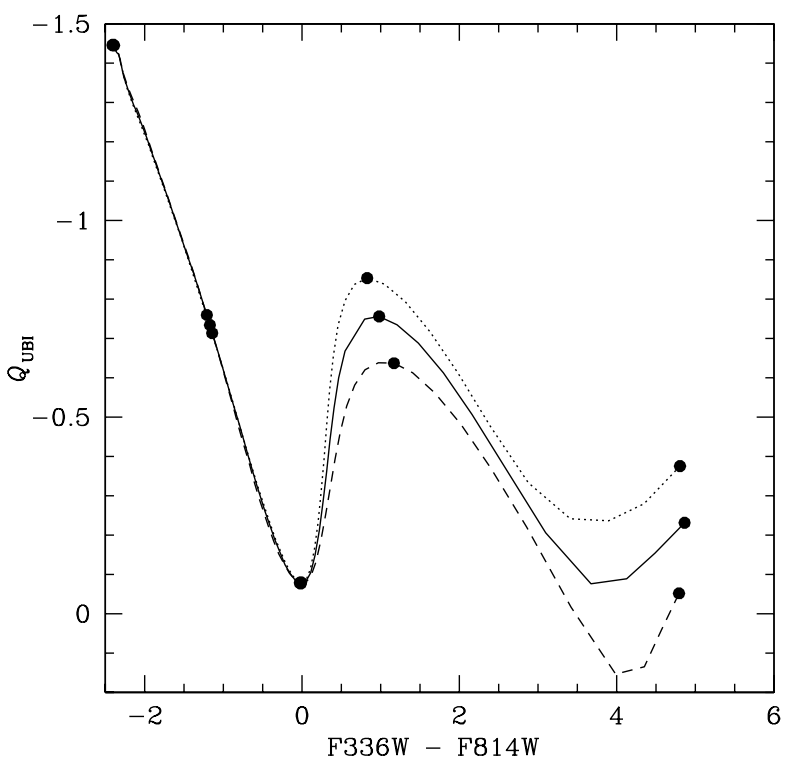

FIG. 16.-Zero-reddening $Q_{U B I}$ vs. F336W-F814W relation for three values of metallicity: $Z=Z_{\odot}$ (dashed line), $Z=0.3 Z_{\odot}$ (continuous line), and $Z=Z_{\odot} / 10$ (dotted line). The model atmospheres are those of Bessel et al. (1998) for $\log g=4.5$. The points for $T_{\text {eff }}=50,000,15,000,9250,6000$, and $3500 \mathrm{~K}$ are marked on each curve.

tions in more than one UV band. Qualitatively, the reason for this is that shorter wavelengths are more affected by interstellar reddening, and small differences in $E(B-V)$ along different lines of sight may result in large differences in the observed flux, thus making the ultraviolet flux of a moderately reddened hot star almost indistinguishable from that of an unreddened, colder one. Quantitatively, the situation is illustrated in Figure 20, where we plot the $1 \sigma$ contour levels for artificial stars of different effective temperature as observed through four optical filters (F439W, F555W, F675W and F814W) and one UV one: F170W (dot-dashed line), F255W (dashed line), or F336W (continuous line). The contours, computed assuming the same photometric error

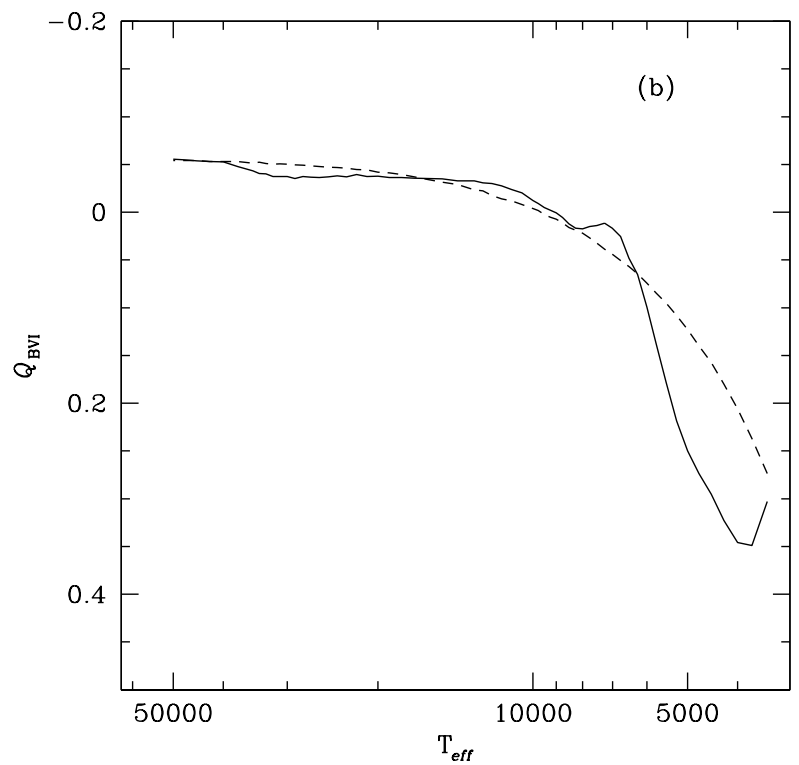

FIG. 17. $-Q_{B V I}$ reddening-free color as a function of F439W-F814W (left $)$ and $T_{\text {eff }}(r i g h t)$ for the Bessel et al. (1998) model atmospheres for $Z=0.3 Z_{\odot}$ and $\log g=4.5$. The case of blackbody spectra is shown in both panels as a dashed line. 

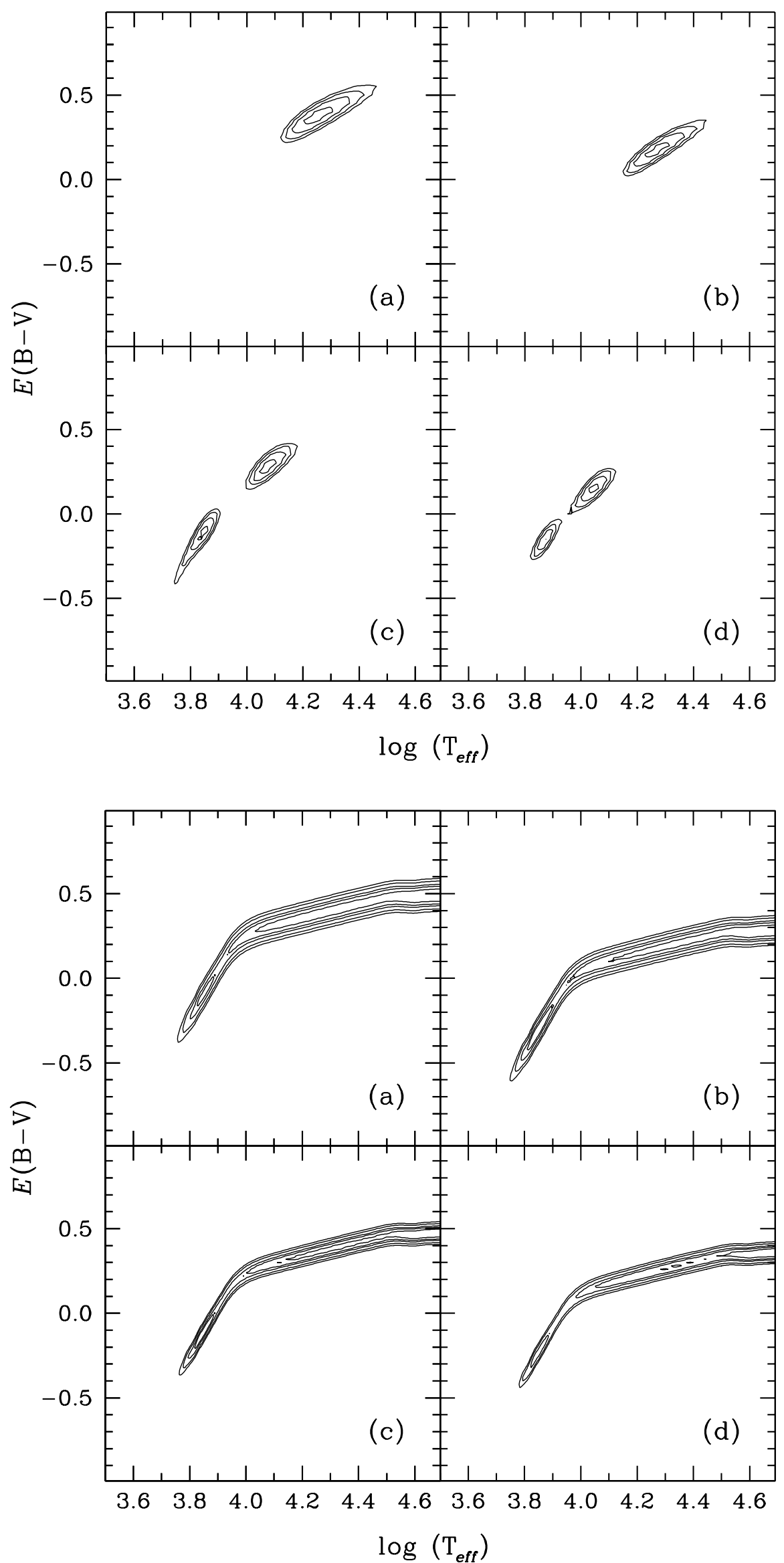

FIG. 18. - Contour plots in the $T_{\text {eff }} E(B-V)$ plane for four stars with unambiguous solutions. Top, only four bands (F439W, F555W, F675W, and F814W) used in the fit; bottom, all six bands used. In both panels the contours are for $\delta \chi^{2}=2$ from the minimum. 

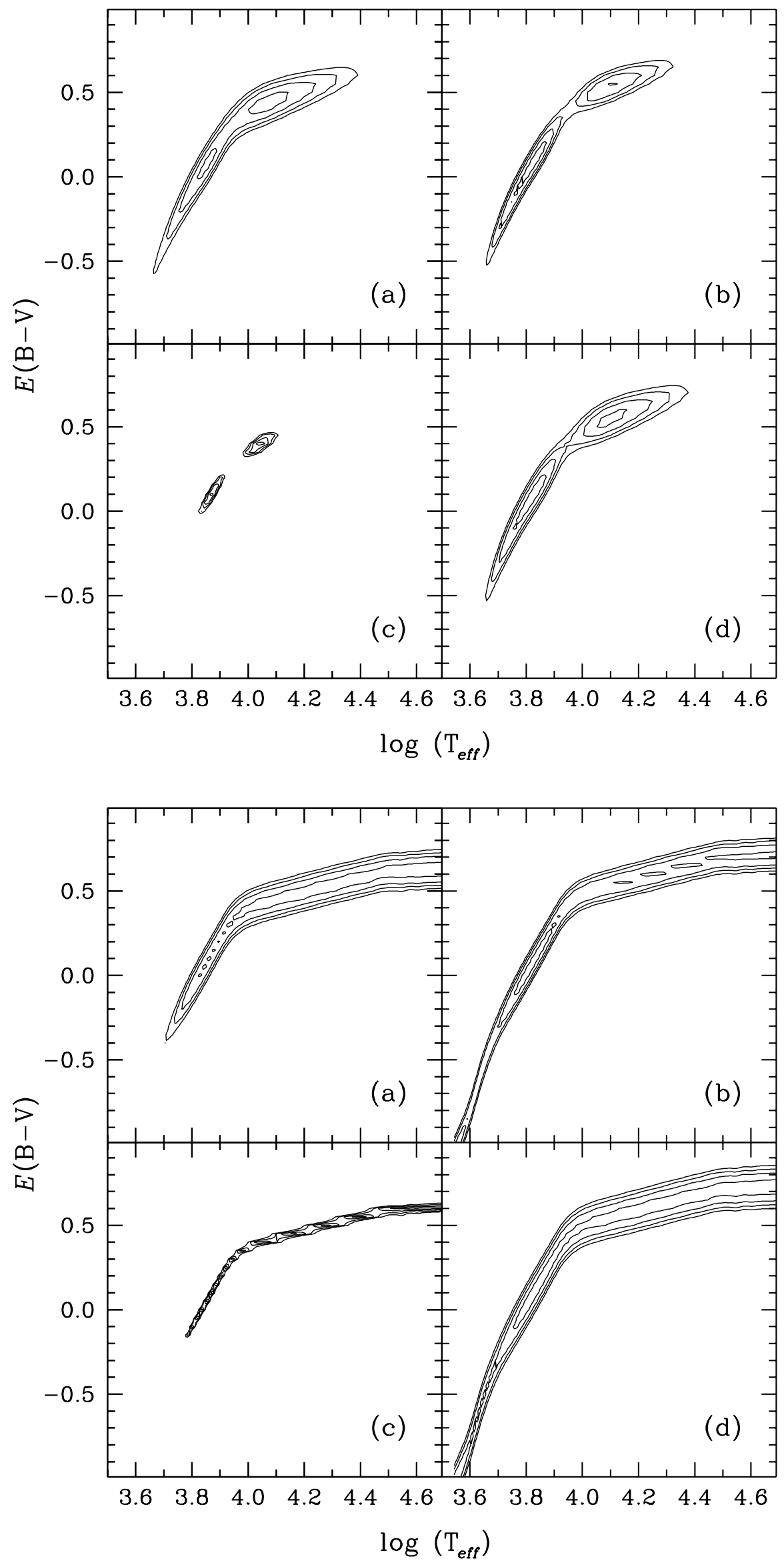

FIG. 19.- Same as Fig. 18, but for stars with multiple solutions 


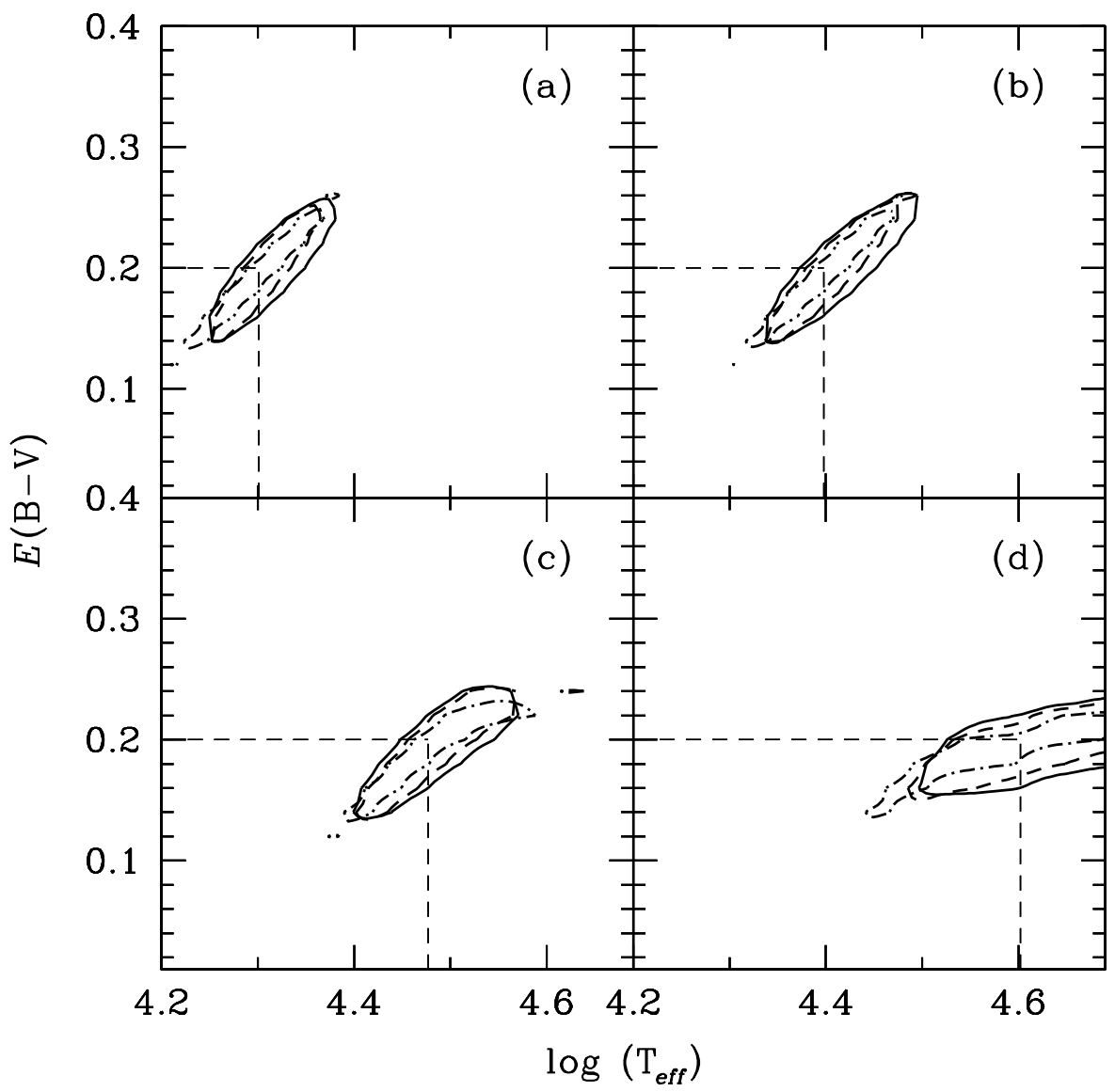

FIG. 20. $-\chi^{2}$ contour map for artificial stars with $E(B-V)=0.2$ and $T_{\text {eff }}=20,000 \mathrm{~K}(a), 25,000 \mathrm{~K}(b), 30,000 \mathrm{~K}(c)$, and 40,000 K $(d)$. The contours correspond to the $1 \sigma$ confidence level for a five-band fit. In all cases the same four optical filters are used (F439W $[B], \mathrm{F} 555 \mathrm{~W}[V], \mathrm{F} 675 \mathrm{~W}[R]$, and F814W $[I])$ in combination with different UV passbands: F170W (dot-dashed line), F255W (dashed line) or F336W (continuous line). The photometric error is assumed to be the same in all three UV filters. The straight dashed lines mark the input parameters of the model stars.

in all three UV filters, overlap almost perfectly. This means that one does not gain in accuracy by observing at shorter wavelengths, even for hot stars. Since the sensitivity of normal CCDs and the emission of cold stars both drop dramatically, the further one moves to the ultraviolet, a filter in the region of the $U$ band is the ideal choice for the filter blueward of the Balmer jump.

As we have pointed out at the beginning of this section, there are three unknowns in the fit, so that six bands are actually redundant and four could be enough to cover the wide range of temperatures spanned by stars in regions with mixed stellar populations. In fact, the method works rather well also with only four bands, provided that they encompass the Balmer jump. This is shown in Figure 21, where the $\mathrm{H}-\mathrm{R}$ diagram for the control field located $8^{\prime}$ southeast of SN $1987 \mathrm{~A}$, derived following our procedure, is shown. In this case, as reported in Table 2, the four available photometric bands were F300W, F450W, F675W, and F814W. The star symbol shows the location of the brightest star in the WFPC2 field of view. It is so heavily saturated in the WFPC2 chip that its image was spilling over several neighboring stars, thus making impossible to make direct measurements, and, therefore, its photometry is taken from Fitzpatrick (1988).

As it can be seen, the observed main sequence, although still in very good agreement with the theoretical one, is less sharply defined than the one in the field of SN 1987A (see Fig. 9), and more stars fall under the theoretical zero-age

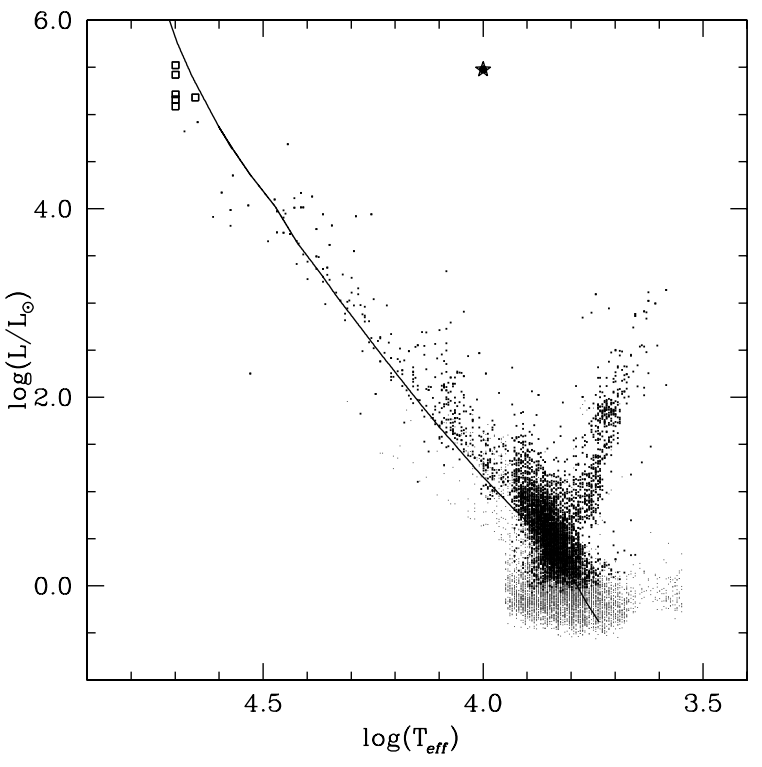

FIG. 21.-H-R diagram for the 13,098 stars detected in the control field for SN 1987A, showing 4912 stars with $\delta \log \left(T_{\text {eff }}\right)<0.05$ (black dots) and stars for which the F300W magnitude is ill determined because of saturation (squares), and hence, the fit was performed excluding this filter. The location of Sk $-69^{\circ} 211$ according to the photometry by Fitzpatrick (1988) is shown with a star symbol. The zero-age main sequence for $Z=0.3 Z$ 。 from the models by Brocato \& Castellani (1993) and Cassisi et al. (1994) for masses below $25 M_{\odot}$ and by Schaerer et al. (1993) above $25 M_{\odot}$ is shown as a continuous line. 
main sequence. Using a reduced set of filters has affected the result in two ways. First, there is only one band blueward of the Balmer jump (F300W) instead of two (F255W and $\mathrm{F} 336 \mathrm{~W}$ ): the fit is less sensitive to temperature for hot stars and, hence, for them the results are less accurate. Second, since the observations were taken as "parallels" to FOS primaries, we did not have enough time to make observations both in the F555W (or a similar band such as F606W) and in the F675W filter. We opted for this latter one to identify stars with $\mathrm{H} \alpha$ emission more accurately. As a result, though, the photometric points are not spaced in wavelength quite evenly. As a consequence, the fit for the cooler stars, the ones that are not well exposed in F300W and F450W bands, has to rely on a short-wavelength baseline and, thus, is less precise than it would have been if observations at roughly $5000 \AA$ had been available. This also results in a larger fraction of stars below the main sequence (roughly 600 out of a total of nearly 13,100, i.e., $4.6 \%$ ) when compared with the main field (roughly 600 out of almost 22,000, i.e., less than 3\%). In general, however, the resulting stellar parameters are still of very good quality, with more than $30 \%$ of the stars with an error in $T_{\text {eff }}$ smaller than $10 \%$.

\section{EFFECT OF RANDOM AND SYSTEMATIC PHOTOMETRIC ERRORS}

To assess the intrinsic precision of the procedure described in this paper we have tested it on simulations. Artificial stars were created with $E(B-V)=0.2$ in the six filters listed in Table 1, evenly distributed in the logarithm of effective temperature between 3500 and $50,000 \mathrm{~K}$, the range covered by Bessel et al. (1998) models. They were then fitted according to the prescriptions of $\S 4$.

\subsection{Random Errors}

The effects of random errors were mimicked by creating three groups of 10,000 stars and adding to the model magnitudes errors drawn from Gaussians of different rms $(0.02$, 0.05 , and $0.1 \mathrm{mag}$ ). These stars were then fitted using only four or five bands or the complete set of six bands.

The situation for a five-band fit $(\mathrm{F} 336 \mathrm{~W}, \mathrm{~F} 439 \mathrm{~W}$, F555W, F675W, and F814W) with varying photometric precision is shown in Figure 22. There we plot the ratio of the output to input temperature as a function of the input temperature for different values of the error rms: $(a) 0.1,(b)$ 0.05 , and (c) $0.02 \mathrm{mag}$. In each panel the photometric errors were drawn from the same Gaussian, independent of the star's temperature. As it can be seen, there is no systematic trend, and the points are mostly scattered around $T_{\text {out }}=$

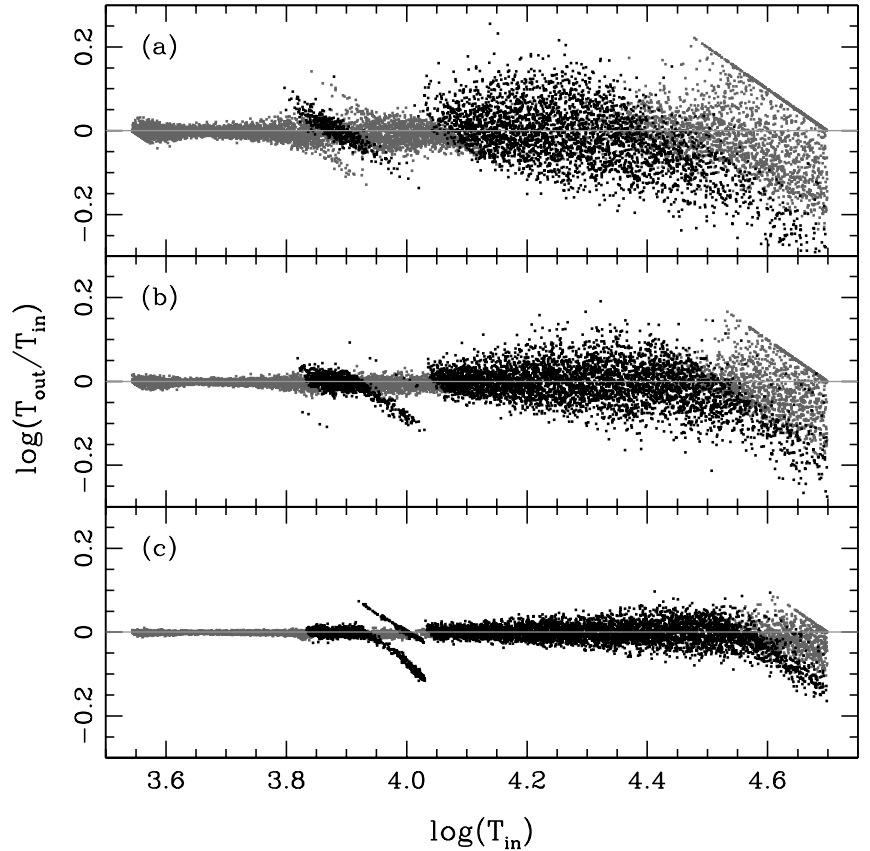

FIG. 22.- Ratio of output to input temperature as a function of the latter for 10,000 model stars distributed evenly in $\log \left(T_{\text {in }}\right)$. The fit was performed as described in $\S 4$ using five bands: F336W, F439W, F555W, F675W, and F814W. The input stars in $(a)$ simulate a random photometric error of 0.1 mag in all the bands, those in (b) an error of $0.05 \mathrm{mag}$, and those in $(c)$ an error of $0.02 \mathrm{mg}$. Black dots represent class I and II stars for which the reddening was determined individually, while gray ones represent class III stars dereddened with the average value of their class I and II neighbors.

$T_{\text {in }}$. The values of the mean and the dispersion are reported in Table 5.

The biggest deviation from the random scatter is represented by the two features at $\log \left(T_{\text {in }}\right) \simeq 3.95$, which are most prominent in $(c)$. These are stars close to the turning point at $\mathrm{F} 336 \mathrm{~W}-\mathrm{F} 814 \mathrm{~W} \simeq 0$ in Figure 6 and were assigned the wrong value of $E(B-V)$ during the selection process, which constitutes the first step of the procedure. Note that this effect depends on the input photometric error because the class assignment is based on it. In the worst case, when the error is $0.02 \mathrm{mag}$, however, this systematic error affects less than $10 \%$ of the stars at that temperature, split almost evenly between stars with too high and too low an output temperature. This effect is negligible for studies of stellar populations. However, if one is dealing with special categories of objects, for example, variable or otherwise peculiar stars, which represent a small fraction of the total population in the $3.92 \lesssim \log \left(T_{\text {eff }}\right) \lesssim 4$ range, one should be aware

TABLE 5

Mean Ratio and rms Scatter of the Output-to-Input Values of Selected Quantities for 10,000 Artificial Stars

\begin{tabular}{|c|c|c|c|c|c|c|c|}
\hline \multirow{2}{*}{$\begin{array}{l}\text { INPUT ERROR } \\
\quad(\mathrm{rms})\end{array}$} & \multirow[b]{2}{*}{ FitTed BANDS } & \multicolumn{2}{|c|}{$T_{\text {eff }}$} & \multicolumn{2}{|c|}{$E(B-V)$} & \multicolumn{2}{|c|}{$L$} \\
\hline & & $T_{\text {in }}<9000$ & $T_{\text {in }}>9000$ & $T_{\text {in }}<9000$ & $T_{\text {in }}>9000$ & $T_{\text {in }}<9000$ & $T_{\text {in }}>9000$ \\
\hline $0.1 \ldots \ldots \ldots \ldots \ldots$ & 5 & $0.99 \pm 0.05$ & $0.97 \pm 0.18$ & $0.01 \pm 0.02$ & $-0.02 \pm 0.06$ & $0.98 \pm 0.17$ & $1.01 \pm 0.58$ \\
\hline $0.05 \ldots \ldots \ldots \ldots \ldots$ & 5 & $0.99 \pm 0.03$ & $0.98 \pm 0.12$ & $0.01 \pm 0.02$ & $-0.01 \pm 0.04$ & $0.97 \pm 0.10$ & $0.99 \pm 0.37$ \\
\hline $0.02 \ldots \ldots \ldots \ldots \ldots$ & 5 & $0.99 \pm 0.04$ & $0.99 \pm 0.06$ & $0.01 \pm 0.03$ & $-0.00 \pm 0.02$ & $0.97 \pm 0.11$ & $0.97 \pm 0.18$ \\
\hline $0.05 \ldots \ldots \ldots \ldots \ldots$ & 4 & $0.99 \pm 0.04$ & $0.98 \pm 0.12$ & $0.01 \pm 0.02$ & $-0.01 \pm 0.04$ & $0.98 \pm 0.11$ & $0.99 \pm 0.38$ \\
\hline $0.05 \ldots \ldots \ldots \ldots \ldots$ & 6 & $1.00 \pm 0.03$ & $0.98 \pm 0.11$ & $0.01 \pm 0.02$ & $-0.01 \pm 0.04$ & $0.99 \pm 0.10$ & $0.97 \pm 0.35$ \\
\hline
\end{tabular}

NoTE.-See text. 
that random errors may create false outliers, with temperatures deviating as much as $20 \%$ for up $10 \%$ of the stars in this temperature range. For this type of studies it is safer not to determine extinction for individual stars but, rather, to adopt the value of the neighbors not affected by this problem.

It is interesting to note in all the panels in Figure 22 that the dispersion increases for hotter stars. This is because broadband colors become progressively more insensitive to temperature as it increases. In fact, the scatter at high temperatures becomes smaller when the F255W filter is added to the fit, as shown in Figure 23. Of course, in reality hotter main-sequence stars are also brighter than colder ones and thus have smaller photometric errors, which compensate for the lower sensitivity of colors to high effective temperatures. Again, see Table 5 for the values of the mean and dispersion.

\subsection{Systematic Errors}

In addition to random errors that vary from star to star and from band to band, the measured fluxes are also affected by systematic errors in the calibrations of each band, i.e., errors in the photometric zero points. These, of course, are different from band to band but the same for all the stars in each band. Again, we have explored three cases $(0.02,0.05$, and $0.1 \mathrm{mag})$, and the results are shown in Figure 24 , which displays the distributions of the temperature

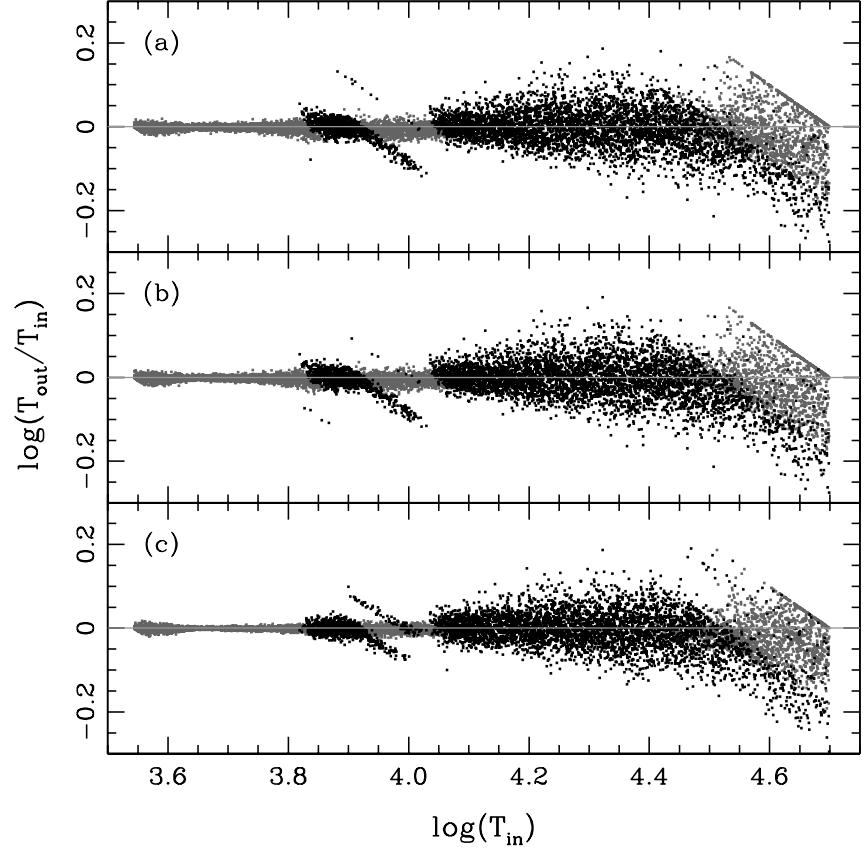

FIG. 23.-Same as Fig. 22, but the fit was performed with different combinations of bands: (a) four bands (F336W, F439W, F555W, and F814W), (b) five bands, those of $(a)$ plus F675W, and $(c)$ six bands, those of $(b)$ plus F255W. The stars simulate a random photometric error of 0.05 mag.

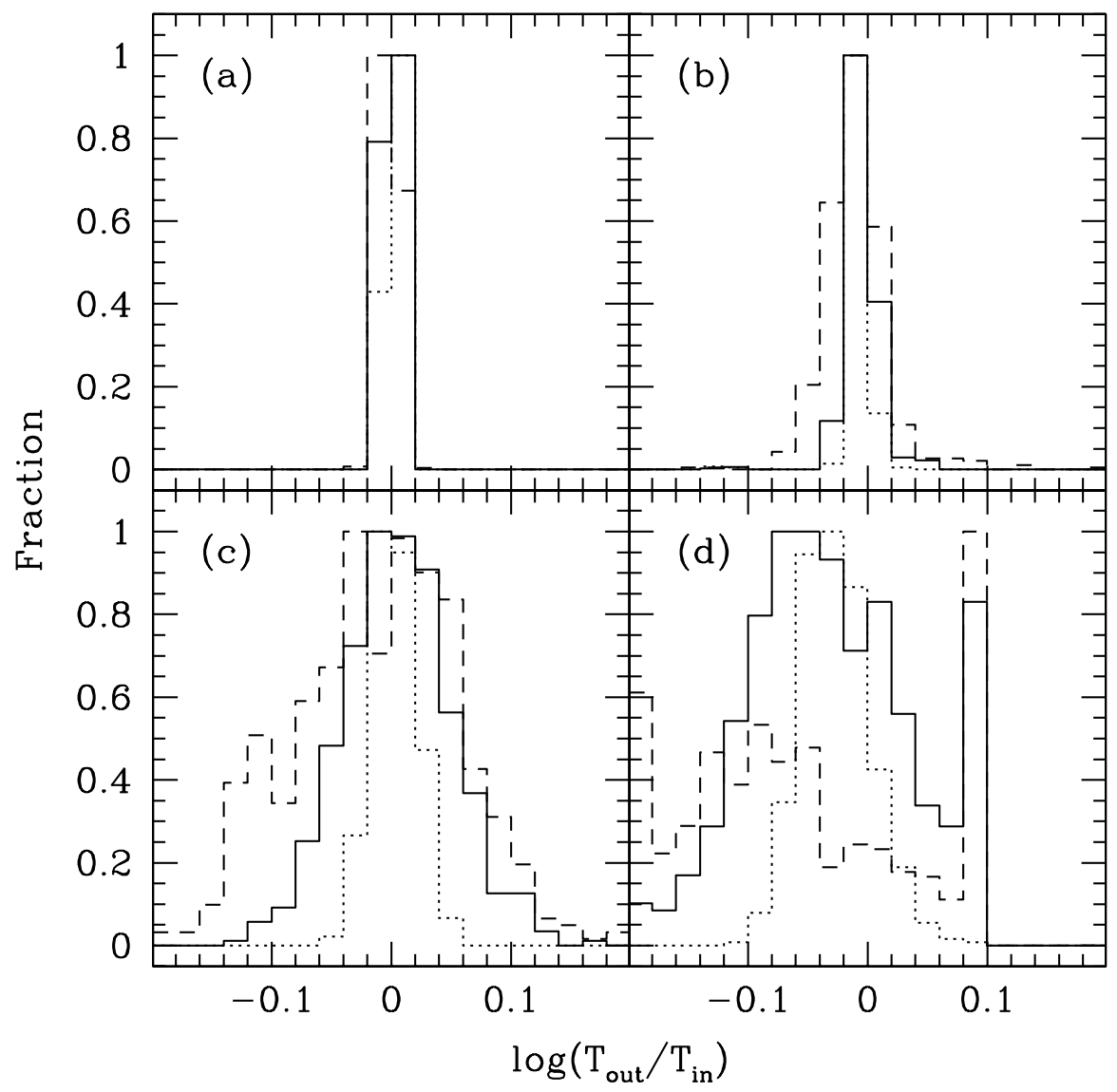

FIG. 24.-Distribution of the ratio of output to input temperature in the case of systematic errors for four representative temperatures: (a) $5000 \mathrm{~K}$, $(b)$ $10,000 \mathrm{~K},(c) 20,000 \mathrm{~K}$, and $(d) 40,000 \mathrm{~K}$. In each panel the dotted line is for the case of a systematic error of 0.02 mag, the continuous one for 0.05 mag, and the dashed one for $0.1 \mathrm{mag}$. 
deviations $\log \left(T_{\text {out }} / T_{\text {in }}\right)$ for four representative temperatures, i.e., 5000, 10,000, 20,000, and 40,000 K.

From an inspection of Figure 24 one notes again that, given the same error, the temperature measured for hot stars is intrinsically more uncertain than for cold ones, with the dispersion growing from less than $1 \%$ for stars at $5000 \mathrm{~K}$, to as much as $12 \%$ at $40,000 \mathrm{~K}$. The sharp peak at $\log \left(T_{\text {out }} /\right.$ $\left.T_{\text {in }}\right) \simeq 0.1$ found for $T_{\text {in }}=40,000 \mathrm{~K}$ (Fig. $24 d$ ) is populated by stars for which the fit, starting from $T_{\text {in }}=40,000 \mathrm{~K}$, would indicate a temperature higher than $50,000 \mathrm{~K}$, the highest in the Bessel et al. (1998) grid. Even though the peak is just an artifact, it is clear that calibration errors much smaller than $0.1 \mathrm{mag}$ are necessary for the data to be useful at all temperatures.

\section{SUMMARY AND CONCLUSIONS}

In this paper we have presented and discussed a new technique that uses multiband photometry to measure reddening, effective temperature, and, given the distance, bolometric luminosity for resolved stars. The method is based on broadband optical and near-ultraviolet photometry and, in the test case presented here for the field around SN 1987A in the Large Magellanic Cloud, yields to a reddening determination every $13 \operatorname{arcsec}^{2}$ : a fine grid indeed! Being able to measure the reddening to thousands of individual stars is of great importance in all the astrophysical environments in which the interstellar medium is clumpy and therefore produces differential reddening, as is the case, for example, of virtually every star-forming region.

There are three unknown quantities in the fit: the radius, the effective temperature, and the reddening. Formally, then, at least three photometric points for each star are required to make the problem well defined, and more are needed when dealing with a stellar population encompassing a wide range of temperatures. Obviously, the more bands are available, the better the result of the fit. As we have discussed thoroughly, at least one of the bands has to fall at wavelengths shorter than the Balmer jump because the optical fluxes alone are not enough to reliably recover both $T_{\text {eff }}$ and $E(B-V)$. On the other hand, though, in the case in which observing time constraints limit to one the number of bands that can be used for UV observations, it is not necessary nor advisable to resort to bands at extremely short wavelengths, and observing just blueward of the Balmer jump, a $U$-like band, is the best choice. Of course, observing in more than one ultraviolet band can only help in reducing the errors on the derived quantities. Although we have used the WFPC2 counterparts to the classical Johnson-Cousin passbands to illustrate our method, it proved to work almost equally well when broader filters were used. Broader filters mean more photons detected per unit time and, ultimately, a more efficient use of the telescope time at one's disposal.

Once the stars are accurately placed in the HertzprungRussell diagram, the physical properties of the stellar population, such as star formation history, mass function, etc., can be studied in detail. In this respect, let us stress again the importance of determining the reddening from the same stars that are the ultimate target of the investigation, without having to resort to uncontrollable assumptions on the magnitude and distribution of the interstellar extinction.

To conclude, we refer the reader to Panagia et al. (2000) for a discussion of the properties of the young stellar popu- lation in the field around SN 1987A and to Romaniello et al. (2000) for an application of the dereddened color-magnitude diagram to measuring the distance to the Large Magellanic Cloud. More work on the derivation of the initial mass function and the star formation rate in the SN 1987A field is in progress, and the results will be presented in a forthcoming paper (Romaniello et al. 2002).

It is a pleasure to thank Giuseppe Bertin for many stimulating discussions during the development of the method described here. We gladly acknowledge the comments of an anonymous referee, which helped us greatly to improve the paper, both in the contents and the presentation. M. R. and S. S. acknowledge the kind hospitality of STScI during a number of stays. Support for the SINS program was provided by NASA through grant GO-9114 from the Space Telescope Science Institute, which is operated by the Association of Universities for Research in Astronomy, Inc., under NASA contract NAS-26555. This work was supported in part by STScl-DDRF grants 82131, 82160, and 82186 to N.P.

\section{APPENDIX A \\ BROADBAND COLORS AND EXTINCTION COEFFICIENTS IN SELECTED WFPC2 FILTERS}

The WFPC2 is equipped with a large set of broad-, medium-, and narrowband filters for imaging (see Biretta et al. 2000 for a complete list and full details). Some of them closely match filters in the standard Johnson-Cousin (e.g., the F555W and the $V$, the F439W and the $B$, etc.) or Strömgren (e.g., the F410M and the $v$, the F467M and the $b$, etc.) systems, while some others have no direct counterpart in any standard ground-based set, e.g., the F450W and F606W, to name just two, or, obviously, all ultraviolet passbands.

Color transformations have been devised to convert the WFPC2 magnitudes to the corresponding ones in the Johnson-Cousin system, but they are limited to only a handful of passbands. Moreover, quoting directly from Biretta et al. (2000), they " should be used with caution for quantitative work," because they are just first-order approximations and unavoidably degrade the quality of the original photometry. A much better option, when comparing WFPC2 photometry with theoretical expectations, is to compute these latter ones directly in the passbands used in the observations. As explained in $\S 4$, this is what we have done in this paper, thus minimizing the uncertainties in the comparison between the observed WFPC2 magnitudes and the synthetic ones computed using the Bessel et al. (1998) models.

In Tables 6 and 7 we list the magnitudes in widely used WFPC2 passbands for solar metallicity and for $[\mathrm{Fe} / \mathrm{H}]=-0.5$, the appropriate value for the LMC, respectively. They were computed using the IRAF SYNPHOT synthetic photometry package, and for the sake of generality the magnitudes in the ultraviolet filters are referred to the decontaminated camera. The throughput degradation due to the accumulation of material between decontamination can be easily computed with the formulae given in Bagget et al. (1996). The magnitudes are for a $1 R_{\odot}$ star as seen from a 
TABLE 6

Magnitudes in Selected WFPC2 Broadband Filters

\begin{tabular}{|c|c|c|c|c|c|c|c|c|c|c|}
\hline$T_{\text {eff }}$ & F170W & $\mathrm{F} 255 \mathrm{~W}$ & F300W & F336W & F439W & F450W & F555W & F606W & F675W & F814W \\
\hline \multicolumn{11}{|c|}{$\log g=3$} \\
\hline $3500 \ldots \ldots \ldots$ & 11.930 & 16.528 & 12.843 & 12.115 & 10.320 & 10.094 & 8.969 & 8.566 & 8.115 & 7.003 \\
\hline $4000 \ldots \ldots \ldots$ & 10.692 & 14.882 & 11.338 & 10.494 & 8.817 & 8.407 & 7.308 & 6.864 & 6.399 & 5.835 \\
\hline $4500 \ldots \ldots \ldots$ & 9.900 & 12.670 & 9.480 & 8.604 & 7.604 & 7.189 & 6.334 & 5.968 & 5.570 & 5.160 \\
\hline $5000 \ldots \ldots \ldots$ & 9.250 & 9.935 & 7.882 & 7.172 & 6.652 & 6.305 & 5.614 & 5.319 & 4.990 & 4.662 \\
\hline $5500 \ldots \ldots \ldots$ & 8.665 & 8.278 & 6.710 & 6.122 & 5.860 & 5.600 & 5.051 & 4.809 & 4.533 & 4.265 \\
\hline $6000 \ldots \ldots \ldots$ & 8.085 & 7.105 & 5.850 & 5.351 & 5.196 & 5.008 & 4.585 & 4.390 & 4.162 & 3.945 \\
\hline $6500 \ldots \ldots \ldots$ & 7.381 & 6.183 & 5.207 & 4.793 & 4.629 & 4.497 & 4.180 & 4.029 & 3.847 & 3.678 \\
\hline 7000 ........... & 6.501 & 5.442 & 4.712 & 4.381 & 4.142 & 4.051 & 3.820 & 3.709 & 3.573 & 3.449 \\
\hline $7500 \ldots \ldots \ldots . .$. & 5.647 & 4.829 & 4.295 & 4.040 & 3.707 & 3.652 & 3.502 & 3.430 & 3.340 & 3.255 \\
\hline $8000 \ldots \ldots \ldots$ & 4.836 & 4.274 & 3.898 & 3.711 & 3.338 & 3.312 & 3.234 & 3.195 & 3.144 & 3.091 \\
\hline $8500 \ldots \ldots \ldots$ & 4.077 & 3.745 & 3.498 & 3.375 & 3.057 & 3.045 & 3.018 & 3.000 & 2.977 & 2.947 \\
\hline $9000 \ldots \ldots \ldots$ & 3.415 & 3.278 & 3.128 & 3.058 & 2.844 & 2.840 & 2.849 & 2.844 & 2.837 & 2.824 \\
\hline $9500 \ldots \ldots \ldots$ & 2.886 & 2.869 & 2.790 & 2.762 & 2.675 & 2.674 & 2.706 & 2.709 & 2.713 & 2.712 \\
\hline $10000 \ldots \ldots$ & 2.456 & 2.503 & 2.474 & 2.479 & 2.532 & 2.532 & 2.580 & 2.587 & 2.598 & 2.608 \\
\hline $10500 \ldots \ldots$. & 2.089 & 2.178 & 2.184 & 2.212 & 2.405 & 2.406 & 2.465 & 2.476 & 2.491 & 2.509 \\
\hline $11000 \ldots \ldots$ & 1.768 & 1.886 & 1.919 & 1.965 & 2.291 & 2.293 & 2.360 & 2.373 & 2.391 & 2.418 \\
\hline $11500 \ldots \ldots$. & 1.480 & 1.622 & 1.680 & 1.742 & 2.186 & 2.189 & 2.264 & 2.279 & 2.300 & 2.333 \\
\hline $12000 \ldots \ldots$. & 1.220 & 1.381 & 1.464 & 1.544 & 2.090 & 2.093 & 2.175 & 2.192 & 2.217 & 2.256 \\
\hline $12500 \ldots \ldots$. & 0.981 & 1.161 & 1.268 & 1.367 & 2.000 & 2.004 & 2.093 & 2.112 & 2.140 & 2.185 \\
\hline $13000 \ldots \ldots$. & 0.762 & 0.959 & 1.091 & 1.208 & 1.916 & 1.921 & 2.016 & 2.038 & 2.069 & 2.119 \\
\hline $15000 \ldots \ldots$. & 0.034 & 0.290 & 0.505 & 0.685 & 1.622 & 1.630 & 1.747 & 1.778 & 1.821 & 1.889 \\
\hline $17000 \ldots \ldots$ & -0.537 & -0.242 & 0.034 & 0.263 & 1.367 & 1.376 & 1.513 & 1.551 & 1.606 & 1.690 \\
\hline $19000 \ldots \ldots$. & -1.016 & -0.695 & -0.374 & -0.108 & 1.135 & 1.145 & 1.296 & 1.341 & 1.406 & 1.503 \\
\hline $21000 \ldots \ldots$ & -1.441 & -1.091 & -0.737 & -0.445 & 0.921 & 0.930 & 1.091 & 1.141 & 1.212 & 1.320 \\
\hline $23000 \ldots \ldots$ & -1.832 & -1.435 & -1.053 & -0.741 & 0.724 & 0.733 & 0.904 & 0.956 & 1.032 & 1.147 \\
\hline $25000 \ldots \ldots$. & -2.197 & -1.737 & -1.337 & -1.008 & 0.537 & 0.546 & 0.726 & 0.782 & 0.863 & 0.985 \\
\hline $27000 \ldots \ldots$ & -2.492 & -1.918 & -1.494 & -1.143 & 0.395 & 0.410 & 0.605 & 0.670 & 0.763 & 0.896 \\
\hline \multicolumn{11}{|c|}{$\log g=4.5$} \\
\hline $3500 \ldots \ldots \ldots$ & 11.574 & 15.806 & 12.299 & 11.493 & 10.049 & 9.738 & 8.511 & 8.041 & 7.543 & 6.645 \\
\hline $4000 \ldots \ldots \ldots$ & 10.653 & 14.317 & 11.056 & 10.202 & 8.763 & 8.418 & 7.303 & 6.847 & 6.373 & 5.793 \\
\hline $4500 \ldots \ldots \ldots$ & 9.904 & 12.589 & 9.514 & 8.630 & 7.584 & 7.239 & 6.357 & 5.979 & 5.567 & 5.155 \\
\hline $5000 \ldots \ldots \ldots$ & 9.250 & 9.912 & 7.857 & 7.118 & 6.630 & 6.310 & 5.624 & 5.326 & 4.991 & 4.662 \\
\hline $5500 \ldots \ldots \ldots$ & 8.654 & 8.115 & 6.609 & 6.013 & 5.875 & 5.614 & 5.060 & 4.816 & 4.537 & 4.267 \\
\hline $6000 \ldots \ldots \ldots$ & 8.047 & 6.844 & 5.666 & 5.170 & 5.244 & 5.046 & 4.605 & 4.405 & 4.170 & 3.947 \\
\hline $6500 \ldots \ldots \ldots$ & 7.301 & 5.855 & 4.947 & 4.538 & 4.712 & 4.565 & 4.220 & 4.057 & 3.863 & 3.681 \\
\hline $7000 \ldots \ldots \ldots$ & 6.337 & 5.065 & 4.399 & 4.074 & 4.256 & 4.149 & 3.880 & 3.751 & 3.595 & 3.453 \\
\hline $7500 \ldots \ldots \ldots$ & 5.459 & 4.433 & 3.971 & 3.723 & 3.857 & 3.779 & 3.569 & 3.473 & 3.355 & 3.251 \\
\hline $8000 \ldots \ldots \ldots$ & 4.703 & 3.913 & 3.607 & 3.429 & 3.502 & 3.447 & 3.289 & 3.223 & 3.142 & 3.074 \\
\hline $8500 \ldots \ldots \ldots$ & 3.980 & 3.472 & 3.283 & 3.161 & 3.187 & 3.156 & 3.050 & 3.013 & 2.966 & 2.928 \\
\hline $9000 \ldots \ldots \ldots$ & 3.330 & 3.082 & 2.979 & 2.904 & 2.935 & 2.922 & 2.860 & 2.844 & 2.823 & 2.807 \\
\hline $9500 \ldots \ldots \ldots$. & 2.801 & 2.731 & 2.694 & 2.661 & 2.740 & 2.737 & 2.708 & 2.705 & 2.701 & 2.703 \\
\hline $10000 \ldots \ldots$. & 2.364 & 2.417 & 2.431 & 2.434 & 2.580 & 2.583 & 2.581 & 2.587 & 2.595 & 2.609 \\
\hline $10500 \ldots \ldots$. & 1.999 & 2.128 & 2.182 & 2.215 & 2.443 & 2.449 & 2.468 & 2.480 & 2.497 & 2.521 \\
\hline $11000 \ldots \ldots$ & 1.682 & 1.863 & 1.948 & 2.006 & 2.322 & 2.330 & 2.366 & 2.383 & 2.406 & 2.437 \\
\hline $11500 \ldots \ldots$. & 1.401 & 1.620 & 1.728 & 1.806 & 2.213 & 2.223 & 2.272 & 2.292 & 2.319 & 2.358 \\
\hline $12000 \ldots \ldots$. & 1.146 & 1.396 & 1.524 & 1.618 & 2.114 & 2.124 & 2.183 & 2.206 & 2.237 & 2.282 \\
\hline $12500 \ldots \ldots$. & 0.914 & 1.190 & 1.334 & 1.443 & 2.022 & 2.033 & 2.101 & 2.126 & 2.160 & 2.211 \\
\hline $13000 \ldots \ldots$. & 0.700 & 0.999 & 1.160 & 1.282 & 1.937 & 1.949 & 2.024 & 2.051 & 2.088 & 2.144 \\
\hline $15000 \ldots \ldots$. & -0.016 & 0.361 & 0.582 & 0.756 & 1.644 & 1.658 & 1.758 & 1.793 & 1.841 & 1.913 \\
\hline $17000 \ldots \ldots$. & -0.577 & -0.137 & 0.137 & 0.358 & 1.401 & 1.418 & 1.539 & 1.581 & 1.639 & 1.724 \\
\hline $19000 \ldots \ldots$. & -1.040 & -0.550 & -0.232 & 0.025 & 1.188 & 1.206 & 1.345 & 1.393 & 1.461 & 1.557 \\
\hline $21000 \ldots \ldots$ & -1.440 & -0.909 & -0.557 & -0.270 & 0.991 & 1.011 & 1.165 & 1.220 & 1.295 & 1.403 \\
\hline $23000 \ldots \ldots$. & -1.800 & -1.231 & -0.851 & -0.540 & 0.807 & 0.827 & 0.995 & 1.054 & 1.137 & 1.254 \\
\hline $25000 \ldots \ldots$. & -2.132 & -1.525 & -1.121 & -0.789 & 0.633 & 0.653 & 0.831 & 0.895 & 0.984 & 1.109 \\
\hline $27000 \ldots \ldots$. & -2.446 & -1.792 & -1.367 & -1.018 & 0.468 & 0.488 & 0.676 & 0.743 & 0.839 & 0.971 \\
\hline $29000 \ldots \ldots$. & -2.744 & -2.040 & -1.597 & -1.231 & 0.307 & 0.328 & 0.527 & 0.598 & 0.699 & 0.838 \\
\hline $31000 \ldots \ldots$ & -3.015 & -2.271 & -1.816 & -1.437 & 0.147 & 0.169 & 0.378 & 0.452 & 0.558 & 0.704 \\
\hline $33000 \ldots \ldots$. & -3.238 & -2.472 & -2.012 & -1.625 & -0.002 & 0.020 & 0.237 & 0.313 & 0.423 & 0.572 \\
\hline $35000 \ldots \ldots$. & -3.412 & -2.632 & -2.170 & -1.781 & -0.127 & -0.104 & 0.117 & 0.193 & 0.304 & 0.456 \\
\hline $37000 \ldots \ldots$ & -3.551 & -2.755 & -2.292 & -1.901 & -0.226 & -0.204 & 0.021 & 0.097 & 0.207 & 0.360 \\
\hline $39000 \ldots \ldots$. & -3.666 & -2.851 & -2.386 & -1.993 & -0.304 & -0.281 & -0.053 & 0.023 & 0.132 & 0.286 \\
\hline $41000 \ldots \ldots$ & -3.768 & -2.933 & -2.465 & -2.069 & -0.370 & -0.347 & -0.115 & -0.039 & 0.071 & 0.226 \\
\hline $43000 \ldots \ldots$ & -3.862 & -3.008 & -2.537 & -2.139 & -0.431 & -0.407 & -0.173 & -0.096 & 0.015 & 0.171 \\
\hline
\end{tabular}


TABLE 6-Continued

\begin{tabular}{ccccccccccc}
\hline \hline$T_{\text {eff }}$ & F170W & F255W & F300W & F336W & F439W & F450W & F555W & F606W & F675W & F814W \\
\hline $45000 \ldots \ldots$. & -3.949 & -3.077 & -2.605 & -2.204 & -0.489 & -0.465 & -0.228 & -0.150 & -0.039 & 0.118 \\
$47000 \ldots \ldots$. & -4.030 & -3.142 & -2.668 & -2.266 & -0.543 & -0.519 & -0.281 & -0.202 & -0.090 & 0.067 \\
$49000 \ldots \ldots$. & -4.106 & -3.202 & -2.727 & -2.323 & -0.595 & -0.570 & -0.331 & -0.251 & -0.139 & 0.019 \\
\hline
\end{tabular}

Note.-From the Bessel et al. (1998) models for $Z=Z_{\odot}=0.02$, referred to a $1 R_{\odot}$ star at a distance of 10 pc (see eq. [A1]).

TABLE 7

Magnitudes in Selected WFPC2 Broadband Filters

\begin{tabular}{|c|c|c|c|c|c|c|c|c|c|c|}
\hline$T_{\text {eff }}$ & F170W & F255W & F300W & F336W & F439W & F450W & F555W & F606W & F675W & $\mathrm{F} 814 \mathrm{~W}$ \\
\hline \multicolumn{11}{|c|}{$\log g=3$} \\
\hline $3500 \ldots \ldots \ldots \ldots \ldots$ & 11.592 & 16.214 & 12.581 & 11.833 & 10.214 & 9.855 & 8.598 & 8.116 & 7.605 & 6.648 \\
\hline $4000 \ldots \ldots \ldots \ldots \ldots$ & 10.663 & 14.631 & 11.027 & 10.147 & 8.732 & 8.331 & 7.282 & 6.832 & 6.354 & 5.809 \\
\hline 4500 & 9.877 & 11.619 & 9.048 & 8.245 & 7.504 & 7.120 & 6.323 & 5.973 & 5.585 & 5.166 \\
\hline $5000 \ldots \ldots \ldots \ldots \ldots$ & 9.212 & 9.214 & 7.539 & 6.909 & 6.576 & 6.261 & 5.621 & 5.338 & 5.016 & 4.675 \\
\hline $5500 \ldots \ldots \ldots \ldots \ldots$ & 8.586 & 7.704 & 6.430 & 5.910 & 5.811 & 5.576 & 5.074 & 4.841 & 4.570 & 4.292 \\
\hline $6000 \ldots \ldots \ldots \ldots . . . . . .$. & 7.880 & 6.595 & 5.617 & 5.189 & 5.168 & 5.001 & 4.620 & 4.431 & 4.206 & 3.980 \\
\hline $6500 \ldots \ldots \ldots \ldots . . . .$. & 7.004 & 5.761 & 5.035 & 4.694 & 4.622 & 4.504 & 4.218 & 4.071 & 3.891 & 3.713 \\
\hline 7000 & 6.157 & 5.119 & 4.591 & 4.329 & 4.147 & 4.064 & 3.856 & 3.747 & 3.613 & 3.480 \\
\hline $7500 \ldots \ldots \ldots \ldots \ldots$ & 5.395 & 4.590 & 4.211 & 4.017 & 3.722 & 3.670 & 3.533 & 3.463 & 3.373 & 3.281 \\
\hline 8000 ..................... & 4.637 & 4.114 & 3.848 & 3.711 & 3.354 & 3.331 & 3.263 & 3.225 & 3.174 & 3.114 \\
\hline 8500 & 3.927 & 3.646 & 3.478 & 3.394 & 3.078 & 3.068 & 3.045 & 3.028 & 3.004 & 2.969 \\
\hline 9000 ........................ & 3.318 & 3.214 & 3.123 & 3.085 & 2.870 & 2.865 & 2.875 & 2.869 & 2.861 & 2.844 \\
\hline $9500 \ldots \ldots \ldots \ldots \ldots$ & 2.825 & 2.819 & 2.788 & 2.788 & 2.704 & 2.702 & 2.733 & 2.734 & 2.736 & 2.731 \\
\hline $10000 \ldots \ldots \ldots \ldots$ & 2.414 & 2.465 & 2.479 & 2.508 & 2.565 & 2.564 & 2.609 & 2.614 & 2.622 & 2.628 \\
\hline $11000 \ldots \ldots \ldots \ldots . .$. & 1.748 & 1.872 & 1.945 & 2.013 & 2.331 & 2.332 & 2.396 & 2.406 & 2.422 & 2.444 \\
\hline $12000 \ldots \ldots \ldots \ldots$ & 1.213 & 1.392 & 1.510 & 1.611 & 2.135 & 2.137 & 2.215 & 2.231 & 2.253 & 2.288 \\
\hline $13000 \ldots \ldots \ldots \ldots$ & 0.764 & 0.988 & 1.150 & 1.283 & 1.964 & 1.967 & 2.058 & 2.079 & 2.108 & 2.154 \\
\hline $15000 \ldots \ldots \ldots \ldots$ & 0.039 & 0.339 & 0.574 & 0.765 & 1.671 & 1.677 & 1.791 & 1.820 & 1.862 & 1.927 \\
\hline $17000 \ldots \ldots \ldots \ldots$ & -0.538 & -0.183 & 0.107 & 0.342 & 1.416 & 1.425 & 1.558 & 1.595 & 1.648 & 1.729 \\
\hline $19000 \ldots \ldots \ldots \ldots$ & -1.025 & -0.627 & -0.296 & -0.027 & 1.185 & 1.196 & 1.344 & 1.388 & 1.451 & 1.545 \\
\hline $21000 \ldots \ldots \ldots \ldots$ & -1.452 & -1.016 & -0.655 & -0.360 & 0.972 & 0.983 & 1.144 & 1.193 & 1.262 & 1.367 \\
\hline $23000 \ldots \ldots \ldots \ldots$ & -1.839 & -1.359 & -0.974 & -0.659 & 0.774 & 0.786 & 0.956 & 1.009 & 1.084 & 1.196 \\
\hline $25000 \ldots \ldots \ldots \ldots .$. & -2.199 & -1.670 & -1.266 & -0.934 & 0.583 & 0.594 & 0.775 & 0.831 & 0.912 & 1.032 \\
\hline \multicolumn{11}{|c|}{$\log g=4.5$} \\
\hline $3500 \ldots \ldots \ldots \ldots \ldots$ & 11.438 & 15.667 & 12.222 & 11.409 & 10.067 & 9.678 & 8.397 & 7.860 & 7.299 & 6.488 \\
\hline 4000 & 10.589 & 14.012 & 10.782 & 9.907 & 8.693 & 8.320 & 7.249 & 6.775 & 6.273 & 5.722 \\
\hline $4500 \ldots \ldots \ldots \ldots \ldots$ & 9.883 & 11.779 & 9.151 & 8.312 & 7.496 & 7.163 & 6.342 & 5.979 & 5.575 & 5.158 \\
\hline 5000 & 9.214 & 9.190 & 7.549 & 6.889 & 6.569 & 6.267 & 5.625 & 5.340 & 5.013 & 4.675 \\
\hline $5500 \ldots \ldots \ldots \ldots \ldots$ & 8.570 & 7.566 & 6.353 & 5.824 & 5.839 & 5.595 & 5.079 & 4.844 & 4.569 & 4.290 \\
\hline 6000 ........................ & 7.827 & 6.369 & 5.446 & 5.016 & 5.231 & 5.048 & 4.641 & 4.446 & 4.214 & 3.982 \\
\hline 6500 & 6.882 & 5.421 & 4.729 & 4.378 & 4.743 & 4.602 & 4.275 & 4.113 & 3.916 & 3.722 \\
\hline 7000 & 5.920 & 4.741 & 4.271 & 4.015 & 4.279 & 4.179 & 3.930 & 3.802 & 3.646 & 3.495 \\
\hline $7500 \ldots \ldots \ldots \ldots \ldots$ & 5.158 & 4.190 & 3.881 & 3.698 & 3.885 & 3.810 & 3.615 & 3.519 & 3.400 & 3.288 \\
\hline $8000 \ldots \ldots \ldots \ldots \ldots . .$. & 4.490 & 3.738 & 3.548 & 3.426 & 3.531 & 3.478 & 3.329 & 3.263 & 3.181 & 3.105 \\
\hline $8500 \ldots \ldots \ldots \ldots \ldots$ & 3.827 & 3.355 & 3.251 & 3.175 & 3.217 & 3.186 & 3.084 & 3.046 & 2.998 & 2.954 \\
\hline $9000 \ldots \ldots \ldots \ldots . . . . . . .$. & 3.207 & 3.018 & 2.975 & 2.935 & 2.966 & 2.953 & 2.893 & 2.876 & 2.854 & 2.833 \\
\hline 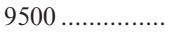 & 2.724 & 2.692 & 2.702 & 2.698 & 2.771 & 2.767 & 2.738 & 2.734 & 2.729 & 2.726 \\
\hline 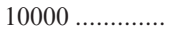 & 2.321 & 2.388 & 2.441 & 2.468 & 2.610 & 2.612 & 2.608 & 2.613 & 2.619 & 2.629 \\
\hline $11000 \ldots \ldots \ldots \ldots$ & 1.673 & 1.853 & 1.966 & 2.042 & 2.356 & 2.363 & 2.395 & 2.410 & 2.430 & 2.458 \\
\hline $12000 \ldots \ldots \ldots \ldots$ & 1.154 & 1.406 & 1.560 & 1.671 & 2.154 & 2.163 & 2.218 & 2.239 & 2.268 & 2.308 \\
\hline $13000 \ldots \ldots \ldots \ldots$ & 0.716 & 1.024 & 1.211 & 1.349 & 1.981 & 1.991 & 2.062 & 2.088 & 2.124 & 2.175 \\
\hline $15000 \ldots \ldots \ldots \ldots$ & 0.005 & 0.404 & 0.646 & 0.833 & 1.690 & 1.703 & 1.800 & 1.834 & 1.880 & 1.949 \\
\hline 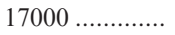 & -0.560 & -0.087 & 0.203 & 0.432 & 1.446 & 1.462 & 1.580 & 1.621 & 1.678 & 1.761 \\
\hline 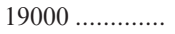 & -1.031 & -0.497 & -0.168 & 0.094 & 1.231 & 1.249 & 1.385 & 1.433 & 1.499 & 1.594 \\
\hline $21000 \ldots \ldots \ldots \ldots$ & -1.439 & -0.854 & -0.494 & -0.204 & 1.034 & 1.054 & 1.206 & 1.259 & 1.333 & 1.438 \\
\hline $23000 \ldots \ldots \ldots \ldots$ & -1.804 & -1.172 & -0.786 & -0.473 & 0.851 & 0.871 & 1.037 & 1.095 & 1.177 & 1.291 \\
\hline $25000 \ldots \ldots \ldots \ldots$ & -2.138 & -1.461 & -1.054 & -0.721 & 0.677 & 0.698 & 0.876 & 0.939 & 1.027 & 1.149 \\
\hline $27000 \ldots \ldots \ldots \ldots . .$. & -2.450 & -1.726 & -1.300 & -0.951 & 0.511 & 0.533 & 0.722 & 0.788 & 0.882 & 1.012 \\
\hline $29000 \ldots \ldots \ldots \ldots \ldots$ & -2.744 & -1.975 & -1.533 & -1.167 & 0.349 & 0.372 & 0.571 & 0.642 & 0.741 & 0.878 \\
\hline $31000 \ldots \ldots \ldots \ldots$ & -3.015 & -2.212 & -1.758 & -1.379 & 0.186 & 0.210 & 0.420 & 0.493 & 0.598 & 0.741 \\
\hline $33000 \ldots \ldots \ldots \ldots$ & -3.242 & -2.425 & -1.964 & -1.578 & 0.031 & 0.055 & 0.273 & 0.349 & 0.457 & 0.605 \\
\hline $35000 \ldots \ldots \ldots \ldots$ & -3.418 & -2.597 & -2.134 & -1.744 & -0.101 & -0.077 & 0.147 & 0.223 & 0.331 & 0.481 \\
\hline $40000 \ldots \ldots \ldots \ldots$ & -3.716 & -2.868 & -2.401 & -2.009 & -0.319 & -0.296 & -0.064 & 0.011 & 0.116 & 0.267 \\
\hline
\end{tabular}

Note.-From the Bessel et al. (1998) models for $Z=0.3 Z_{\odot}=6 \times 10^{-3}$, referred to a $1 R_{\odot}$ star at a distance of $10 \mathrm{pc}$ (see eq. [A1]). 
TABLE 8

Extinction Coefficients $R$ in Selected WFPC2 Broadband Filters

\begin{tabular}{|c|c|c|c|c|c|c|c|c|c|}
\hline$E(B-V)$ & $T_{\text {eff }}=3500 \mathrm{~K}$ & 4000 & 5250 & 6500 & 8750 & 9250 & 10,000 & 25,000 & 40,000 \\
\hline \multicolumn{10}{|c|}{ F170W } \\
\hline $0.0 \ldots \ldots \ldots \ldots$ & 2.017 & 2.205 & 3.060 & 6.202 & 7.901 & 7.956 & 8.009 & 8.110 & 8.111 \\
\hline $0.2 \ldots \ldots \ldots \ldots$ & 1.970 & 2.143 & 2.851 & 5.484 & 7.717 & 7.818 & 7.908 & 8.080 & 8.086 \\
\hline $0.4 \ldots \ldots \ldots \ldots$ & 1.927 & 2.088 & 2.689 & 4.812 & 7.427 & 7.598 & 7.746 & 8.045 & 8.058 \\
\hline $0.6 \ldots \ldots \ldots \ldots$ & 1.889 & 2.039 & 2.559 & 4.265 & 6.993 & 7.248 & 7.480 & 7.998 & 8.023 \\
\hline $0.8 \ldots \ldots \ldots \ldots$ & 1.854 & 1.994 & 2.452 & 3.849 & 6.438 & 6.757 & 7.073 & 7.926 & 7.973 \\
\hline $1.0 \ldots \ldots \ldots \ldots$ & 1.822 & 1.954 & 2.361 & 3.534 & 5.856 & 6.192 & 6.550 & 7.799 & 7.892 \\
\hline $1.2 \ldots \ldots \ldots \ldots$ & 1.793 & 1.916 & 2.284 & 3.289 & 5.331 & 5.649 & 6.003 & 7.578 & 7.745 \\
\hline $1.4 \ldots \ldots \ldots \ldots$ & 1.766 & 1.882 & 2.216 & 3.095 & 4.893 & 5.181 & 5.508 & 7.232 & 7.491 \\
\hline $1.5 \ldots \ldots \ldots \ldots$ & 1.753 & 1.866 & 2.185 & 3.012 & 4.704 & 4.977 & 5.288 & 7.021 & 7.319 \\
\hline \multicolumn{10}{|c|}{$\mathrm{F} 255 \mathrm{~W}$} \\
\hline $0.0 \ldots \ldots \ldots \ldots$ & 4.699 & 5.262 & 6.256 & 6.548 & 6.772 & 6.791 & 6.812 & 6.934 & 6.958 \\
\hline $0.2 \ldots \ldots \ldots \ldots$ & 4.437 & 5.095 & 6.214 & 6.494 & 6.713 & 6.731 & 6.751 & 6.871 & 6.895 \\
\hline $0.4 \ldots \ldots \ldots \ldots$ & 4.147 & 4.892 & 6.171 & 6.443 & 6.657 & 6.675 & 6.696 & 6.813 & 6.836 \\
\hline $0.6 \ldots \ldots \ldots \ldots$ & 3.848 & 4.652 & 6.125 & 6.396 & 6.606 & 6.623 & 6.644 & 6.759 & 6.781 \\
\hline $0.8 \ldots \ldots \ldots \ldots$ & 3.562 & 4.385 & 6.072 & 6.350 & 6.557 & 6.575 & 6.595 & 6.709 & 6.731 \\
\hline $1.0 \ldots \ldots \ldots \ldots$ & 3.304 & 4.108 & 6.005 & 6.303 & 6.510 & 6.528 & 6.549 & 6.662 & 6.684 \\
\hline $1.2 \ldots \ldots \ldots \ldots$ & 3.081 & 3.840 & 5.914 & 6.252 & 6.463 & 6.482 & 6.503 & 6.619 & 6.639 \\
\hline $1.4 \ldots \ldots \ldots \ldots$ & 2.892 & 3.595 & 5.787 & 6.192 & 6.414 & 6.434 & 6.457 & 6.577 & 6.598 \\
\hline $1.5 \ldots \ldots \ldots \ldots$ & 2.809 & 3.483 & 5.707 & 6.156 & 6.388 & 6.408 & 6.433 & 6.557 & 6.578 \\
\hline \multicolumn{10}{|c|}{$\mathrm{F} 300 \mathrm{~W}$} \\
\hline $0.0 \ldots \ldots \ldots \ldots$ & 3.789 & 4.339 & 5.173 & 5.391 & 5.603 & 5.628 & 5.660 & 5.851 & 5.883 \\
\hline $0.2 \ldots \ldots \ldots \ldots$ & 3.577 & 4.185 & 5.131 & 5.353 & 5.558 & 5.582 & 5.614 & 5.804 & 5.836 \\
\hline $0.4 \ldots \ldots \ldots \ldots$ & 3.360 & 4.004 & 5.079 & 5.314 & 5.513 & 5.537 & 5.569 & 5.759 & 5.791 \\
\hline $0.6 \ldots \ldots \ldots \ldots$ & 3.152 & 3.804 & 5.014 & 5.271 & 5.467 & 5.492 & 5.524 & 5.716 & 5.747 \\
\hline $0.8 \ldots \ldots \ldots \ldots$ & 2.965 & 3.598 & 4.931 & 5.221 & 5.419 & 5.444 & 5.477 & 5.674 & 5.705 \\
\hline $1.0 \ldots \ldots \ldots \ldots$ & 2.802 & 3.399 & 4.822 & 5.160 & 5.365 & 5.391 & 5.427 & 5.632 & 5.665 \\
\hline & 2.664 & 3.217 & 4.685 & 5.083 & 5.301 & 5.330 & 5.370 & 5.591 & 5.624 \\
\hline $1.4 \ldots \ldots \ldots \ldots$ & 2.547 & 3.055 & 4.520 & 4.982 & 5.222 & 5.255 & 5.301 & 5.549 & 5.584 \\
\hline $1.5 \ldots \ldots \ldots \ldots$ & 2.496 & 2.982 & 4.429 & 4.921 & 5.175 & 5.211 & 5.260 & 5.526 & 5.563 \\
\hline \multicolumn{10}{|c|}{ F336W } \\
\hline $0.0 \ldots \ldots \ldots \ldots$ & 4.045 & 4.412 & 4.911 & 4.974 & 4.998 & 5.002 & 5.007 & 5.037 & 5.041 \\
\hline $0.2 \ldots \ldots \ldots \ldots$ & 3.888 & 4.296 & 4.888 & 4.962 & 4.990 & 4.994 & 5.000 & 5.033 & 5.037 \\
\hline $0.4 \ldots \ldots \ldots \ldots$ & 3.721 & 4.160 & 4.857 & 4.948 & 4.979 & 4.984 & 4.991 & 5.028 & 5.033 \\
\hline $0.6 \ldots \ldots \ldots \ldots$ & 3.554 & 4.008 & 4.816 & 4.929 & 4.965 & 4.971 & 4.979 & 5.022 & 5.028 \\
\hline $0.8 \ldots \ldots \ldots \ldots$ & 3.397 & 3.848 & 4.761 & 4.903 & 4.947 & 4.954 & 4.964 & 5.016 & 5.022 \\
\hline $1.0 \ldots \ldots \ldots \ldots$ & 3.254 & 3.689 & 4.690 & 4.868 & 4.922 & 4.931 & 4.944 & 5.009 & 5.016 \\
\hline $1.2 \ldots \ldots \ldots \ldots$ & 3.130 & 3.539 & 4.599 & 4.821 & 4.888 & 4.900 & 4.917 & 5.000 & 5.009 \\
\hline $1.4 \ldots \ldots \ldots \ldots$ & 3.023 & 3.403 & 4.489 & 4.759 & 4.842 & 4.858 & 4.880 & 4.988 & 4.999 \\
\hline $1.5 \ldots \ldots \ldots \ldots$ & 2.976 & 3.341 & 4.428 & 4.721 & 4.814 & 4.831 & 4.857 & 4.980 & 4.994 \\
\hline \multicolumn{10}{|c|}{ F439W } \\
\hline $0.0 \ldots \ldots \ldots \ldots$ & 4.088 & 4.097 & 4.132 & 4.152 & 4.161 & 4.163 & 4.166 & 4.178 & 4.181 \\
\hline $0.2 \ldots \ldots \ldots \ldots$ & 4.085 & 4.094 & 4.129 & 4.149 & 4.159 & 4.161 & 4.164 & 4.176 & 4.179 \\
\hline $0.4 \ldots \ldots \ldots \ldots$ & 4.083 & 4.092 & 4.127 & 4.147 & 4.156 & 4.158 & 4.161 & 4.173 & 4.177 \\
\hline $0.6 \ldots \ldots \ldots \ldots$ & 4.080 & 4.089 & 4.124 & 4.144 & 4.154 & 4.156 & 4.159 & 4.171 & 4.174 \\
\hline $0.8 \ldots \ldots \ldots \ldots$ & 4.076 & 4.086 & 4.121 & 4.142 & 4.152 & 4.154 & 4.156 & 4.168 & 4.172 \\
\hline $1.0 \ldots \ldots \ldots \ldots$ & 4.073 & 4.083 & 4.118 & 4.139 & 4.149 & 4.151 & 4.154 & 4.166 & 4.169 \\
\hline $1.2 \ldots \ldots \ldots \ldots$ & 4.069 & 4.079 & 4.116 & 4.136 & 4.147 & 4.149 & 4.151 & 4.163 & 4.167 \\
\hline $1.4 \ldots \ldots \ldots \ldots$ & 4.065 & 4.076 & 4.113 & 4.134 & 4.144 & 4.146 & 4.149 & 4.161 & 4.164 \\
\hline $1.5 \ldots \ldots \ldots \ldots$ & 4.062 & 4.074 & 4.111 & 4.132 & 4.143 & 4.145 & 4.148 & 4.160 & 4.163 \\
\hline \multicolumn{10}{|c|}{ F450W } \\
\hline $0.0 \ldots \ldots \ldots \ldots$ & 3.787 & 3.798 & 3.835 & 3.890 & 3.929 & 3.935 & 3.944 & 3.987 & 4.000 \\
\hline $0.2 \ldots \ldots \ldots \ldots$ & 3.779 & 3.790 & 3.825 & 3.880 & 3.918 & 3.925 & 3.933 & 3.976 & 3.989 \\
\hline $0.4 \ldots \ldots \ldots \ldots$ & 3.772 & 3.782 & 3.816 & 3.869 & 3.908 & 3.914 & 3.922 & 3.965 & 3.977 \\
\hline $0.6 \ldots \ldots \ldots \ldots$ & 3.764 & 3.774 & 3.806 & 3.859 & 3.897 & 3.903 & 3.912 & 3.953 & 3.966 \\
\hline $0.8 \ldots \ldots \ldots \ldots$ & 3.757 & 3.767 & 3.797 & 3.848 & 3.887 & 3.893 & 3.901 & 3.942 & 3.954 \\
\hline $1.0 \ldots \ldots \ldots \ldots$ & 3.749 & 3.759 & 3.789 & 3.838 & 3.876 & 3.882 & 3.890 & 3.930 & 3.942 \\
\hline $1.2 \ldots \ldots \ldots \ldots$ & 3.742 & 3.752 & 3.780 & 3.828 & 3.866 & 3.872 & 3.880 & 3.919 & 3.931 \\
\hline
\end{tabular}


TABLE 8-Continued

\begin{tabular}{|c|c|c|c|c|c|c|c|c|c|}
\hline$E(B-V)$ & $T_{\mathrm{eff}}=3500 \mathrm{~K}$ & 4000 & 5250 & 6500 & 8750 & 9250 & 10,000 & 25,000 & 40,000 \\
\hline $1.4 \ldots \ldots \ldots \ldots$ & 3.735 & 3.745 & 3.771 & 3.818 & 3.856 & 3.862 & 3.869 & 3.908 & 3.919 \\
\hline $1.5 \ldots \ldots \ldots \ldots$ & 3.731 & 3.741 & 3.767 & 3.814 & 3.851 & 3.857 & 3.864 & 3.902 & 3.914 \\
\hline \multicolumn{10}{|c|}{ F555W } \\
\hline $0.0 \ldots$ & 3.031 & 3.066 & 3.153 & 3.189 & 3.243 & 3.249 & 3.255 & 3.281 & 3.288 \\
\hline $0.2 \ldots \ldots \ldots \ldots$ & 3.019 & 3.053 & 3.139 & 3.175 & 3.228 & 3.235 & 3.241 & 3.267 & 3.274 \\
\hline $0.4 \ldots \ldots \ldots \ldots$ & 3.008 & 3.041 & 3.126 & 3.162 & 3.214 & 3.220 & 3.227 & 3.252 & 3.259 \\
\hline $0.6 \ldots \ldots \ldots \ldots$ & 2.997 & 3.030 & 3.113 & 3.148 & 3.200 & 3.206 & 3.212 & 3.238 & 3.245 \\
\hline $0.8 \ldots \ldots \ldots \ldots$ & 2.987 & 3.018 & 3.100 & 3.135 & 3.186 & 3.193 & 3.199 & 3.224 & 3.230 \\
\hline $1.0 \ldots \ldots \ldots \ldots$ & 2.977 & 3.007 & 3.088 & 3.122 & 3.173 & 3.179 & 3.185 & 3.210 & 3.216 \\
\hline $1.2 \ldots \ldots \ldots \ldots$ & 2.967 & 2.997 & 3.076 & 3.110 & 3.159 & 3.166 & 3.172 & 3.196 & 3.203 \\
\hline $1.4 \ldots \ldots \ldots \ldots$ & 2.958 & 2.987 & 3.064 & 3.097 & 3.146 & 3.153 & 3.158 & 3.183 & 3.189 \\
\hline $1.5 \ldots \ldots \ldots \ldots$ & 2.953 & 2.982 & 3.058 & 3.091 & 3.140 & 3.146 & 3.152 & 3.176 & 3.182 \\
\hline \multicolumn{10}{|c|}{ F606W } \\
\hline $0.0 \ldots \ldots \ldots \ldots$ & 2.712 & 2.729 & 2.805 & 2.841 & 2.893 & 2.900 & 2.906 & 2.932 & 2.938 \\
\hline $0.2 \ldots \ldots \ldots \ldots$ & 2.703 & 2.719 & 2.793 & 2.828 & 2.879 & 2.886 & 2.892 & 2.918 & 2.924 \\
\hline $0.4 \ldots \ldots \ldots \ldots$ & 2.694 & 2.709 & 2.781 & 2.815 & 2.866 & 2.873 & 2.879 & 2.904 & 2.910 \\
\hline $0.6 \ldots \ldots \ldots \ldots$ & 2.685 & 2.700 & 2.770 & 2.803 & 2.853 & 2.860 & 2.866 & 2.891 & 2.897 \\
\hline $0.8 \ldots \ldots \ldots \ldots$ & 2.677 & 2.691 & 2.759 & 2.791 & 2.841 & 2.847 & 2.853 & 2.877 & 2.883 \\
\hline $1.0 \ldots \ldots \ldots \ldots$ & 2.669 & 2.682 & 2.748 & 2.780 & 2.828 & 2.834 & 2.840 & 2.864 & 2.870 \\
\hline $1.2 \ldots \ldots \ldots \ldots$ & 2.661 & 2.674 & 2.737 & 2.768 & 2.816 & 2.822 & 2.828 & 2.852 & 2.857 \\
\hline $1.4 \ldots \ldots \ldots \ldots$ & 2.654 & 2.665 & 2.727 & 2.758 & 2.805 & 2.810 & 2.816 & 2.839 & 2.844 \\
\hline $1.5 \ldots \ldots \ldots \ldots$ & 2.650 & 2.661 & 2.722 & 2.752 & 2.799 & 2.805 & 2.810 & 2.833 & 2.838 \\
\hline \multicolumn{10}{|c|}{ F675W } \\
\hline $0.0 \ldots \ldots \ldots \ldots$ & 2.430 & 2.432 & 2.444 & 2.450 & 2.460 & 2.461 & 2.462 & 2.468 & 2.470 \\
\hline $0.2 \ldots \ldots \ldots \ldots$ & 2.427 & 2.429 & 2.441 & 2.447 & 2.457 & 2.458 & 2.460 & 2.465 & 2.467 \\
\hline $0.4 \ldots \ldots \ldots \ldots$ & 2.425 & 2.426 & 2.439 & 2.445 & 2.454 & 2.456 & 2.457 & 2.463 & 2.464 \\
\hline $0.6 \ldots \ldots \ldots \ldots$ & 2.422 & 2.424 & 2.436 & 2.442 & 2.452 & 2.453 & 2.454 & 2.460 & 2.461 \\
\hline $0.8 \ldots \ldots \ldots \ldots$ & 2.419 & 2.421 & 2.433 & 2.439 & 2.449 & 2.450 & 2.451 & 2.457 & 2.459 \\
\hline $1.0 \ldots \ldots \ldots \ldots$ & 2.416 & 2.418 & 2.430 & 2.436 & 2.446 & 2.447 & 2.449 & 2.455 & 2.456 \\
\hline $1.2 \ldots \ldots \ldots \ldots$ & 2.413 & 2.416 & 2.428 & 2.434 & 2.443 & 2.445 & 2.446 & 2.452 & 2.453 \\
\hline $1.4 \ldots \ldots \ldots \ldots$ & 2.411 & 2.413 & 2.425 & 2.431 & 2.441 & 2.442 & 2.443 & 2.449 & 2.451 \\
\hline $1.5 \ldots \ldots \ldots \ldots$ & 2.409 & 2.412 & 2.424 & 2.430 & 2.439 & 2.441 & 2.442 & 2.448 & 2.449 \\
\hline \multicolumn{10}{|c|}{ F814W } \\
\hline $0.0 \ldots \ldots \ldots \ldots$ & 1.786 & 1.829 & 1.860 & 1.875 & 1.892 & 1.894 & 1.896 & 1.912 & 1.916 \\
\hline $0.2 \ldots \ldots \ldots \ldots$ & 1.777 & 1.820 & 1.851 & 1.867 & 1.884 & 1.886 & 1.888 & 1.904 & 1.908 \\
\hline $0.4 \ldots \ldots \ldots \ldots$ & 1.768 & 1.811 & 1.842 & 1.858 & 1.876 & 1.877 & 1.879 & 1.896 & 1.900 \\
\hline $0.6 \ldots \ldots \ldots \ldots$ & 1.759 & 1.801 & 1.833 & 1.849 & 1.867 & 1.868 & 1.870 & 1.888 & 1.892 \\
\hline $0.8 \ldots \ldots \ldots \ldots$ & 1.750 & 1.792 & 1.824 & 1.840 & 1.858 & 1.859 & 1.861 & 1.879 & 1.884 \\
\hline $1.0 \ldots \ldots \ldots \ldots$ & 1.740 & 1.782 & 1.815 & 1.831 & 1.849 & 1.850 & 1.852 & 1.870 & 1.875 \\
\hline $1.2 \ldots \ldots \ldots \ldots$ & 1.731 & 1.773 & 1.805 & 1.822 & 1.839 & 1.841 & 1.843 & 1.861 & 1.866 \\
\hline $1.4 \ldots \ldots \ldots \ldots$ & 1.721 & 1.763 & 1.795 & 1.812 & 1.830 & 1.832 & 1.833 & 1.852 & 1.857 \\
\hline $1.5 \ldots \ldots \ldots \ldots$ & 1.717 & 1.758 & 1.791 & 1.807 & 1.825 & 1.827 & 1.829 & 1.848 & 1.853 \\
\hline
\end{tabular}

Note.-From the Bessel et al. (1998) model atmospheres and the Galactic reddening law of Savage \& Mathis (1979), as a function of effective temperature and $E(B-V)$ (see eq. [A1]).

distance of $10 \mathrm{pc}$, and scaling them for any radius and distance using equation (A1) is straightforward.

The other important quantities that one needs to interpret the data are the extinction coefficients. Again, it is very important to compute them in the very same passbands used for the observations. The values of the normalized extinction $R_{\lambda}=A_{\lambda} / E(B-V)$ for the filters of Tables 6 and 7 computed for the galactic reddening law as compiled by Savage \& Mathis (1979) and the model atmospheres by Bessel et al. (1998) are listed in Table 8 for various values of the effective temperature and the color excess $E(B-V)$.

Using the entries of Tables 6,7 and 8 , then, it is very easy to model a star of metallicity $Z$, surface gravity $g$, effective temperature $T_{\text {eff, }}$, and radius $R$ (in solar units) as seen at a distance $D$ (in parsecs) through a layer of dust characterized by a reddening $E(B-V)$ :

$$
\begin{aligned}
m\left[Z, g, T_{\text {eff }} R, D, E(B-V)\right] & \\
= & m_{0}\left(\mathrm{Z}, g, T_{\text {eff }}\right)-5\left(\frac{R}{1 R_{\odot}}\right)+5 \log \left(\frac{D}{10 \mathrm{pc}}\right) \\
& +R\left(T_{\text {eff }}, E[(E-V)] E(B-V),\right.
\end{aligned}
$$

where $m_{0}\left(Z, g, T_{\text {eff, }} Z\right)$ is the appropriate value, either read or interpolated from Table 6 or 7 , and $R\left[T_{\text {eff }}, E(B-V)\right]$ can be taken from Table 8 . 
Ultraviolet WFPC2 filters are notoriously affected by red leak, a quite substantial one when red stars are observed (see Table 3.13 of Biretta et al. 2000). This is the reason why, for example, as shown in Table 8, the extinction coefficient $R$ in the F170W filter is about 4 times smaller for a $T_{\text {eff }}=3500 \mathrm{~K}$ star than for a $40,000 \mathrm{~K}$ one! One can easily assess the influence of the red leak by recomputing the same coefficients after cutting it out from the response curve of the filter, in this case the F170W. What one finds, then, is that, while the extinction coefficient for $T_{\text {eff }}=40,000 \mathrm{~K}$ is virtually unchanged, the one for $T_{\text {eff }}=3500 \mathrm{~K}$ increases by a factor of 3 , thus reducing the difference between them to about $30 \%$. The residual difference in $R$ between different temperatures, the lower one yielding to lower extinction coefficients, is caused by the fact that filters have a finite, nonnegligible bandwidth, and thus the effective extinction in a band depends on the shape of the spectrum, which is a function of temperature. The same explanation applies for the fact (also illustrated in Table 8) that extinction coefficients become smaller as the optical depth of the dust increases because dust extinction makes a spectral energy distribution become redder. Tables with similar quantities computed for all WFPC2 filters and for all model atmospheres in the Bessel et al. (1998) grid are available and may be provided on request.

\section{APPENDIX B}

\section{FITTING PROCEDURE}

As repeatedly stated, the general idea of our method is to recover simultaneously the extinction along the line of sight and the effective temperature of a star by fitting the observed magnitudes to the ones computed using stellar atmosphere models reddened by various amounts of $E(B-V)$. In this appendix we describe the details of the fitting procedure.

Among the three parameters we want to determine, only two change the shape of the spectrum, i.e., $E(B-V)$ and effective temperature $\left(T_{\text {eff }}\right)$. The third one, i.e., the radius $R$, causes just a rigid shift of the flux on a logarithmic scale. So, we first determine the shape of the spectrum by solving simultaneously for $E(B-V)$ and $T_{\text {eff, }}$ then we compute the radius of the star. For every star, the following steps are performed:

1. The input spectrum is normalized to the band with the highest photometric accuracy, usually the F555W, to eliminate the overall normalization, which depends an the star's radius. Obviously, the theoretical spectra are normalized in the same way. Let $m_{\mathrm{obs}, i}$ be the normalized observed magnitude in filter $i$ and $m_{\mathrm{ms}, i j}^{0}$ the normalized model magnitude for $j$ th temperature in the same filter.

2 . The theoretical magnitudes are reddened for various values of $E(B-V)$. If $R_{i}$ is the extinction coefficient in band $i$ due to the LMC dust and $R_{i, \mathrm{MW}}$ is the one due to our Galaxy,

$$
\begin{aligned}
m_{\mathrm{ms}, i j}[E(B-V)]= & m_{\mathrm{ms}, i j}^{0}+\left[R_{i} E(B-V)\right] \\
& +\left[R_{i, \mathrm{MW}} E(B-V)_{\mathrm{MW}}\right],
\end{aligned}
$$

with $E(B-V)_{\mathrm{MW}}=0.05$, as already discussed. The extinction coefficients for selected WFPC2 filters are listed in Table 8.
3. For any given temperature and reddening, we have computed the $\chi^{2}$ as

$$
\chi_{j}^{2}[E(B-V)]=\sum_{i=1}^{N-1}\left\{\frac{m_{\mathrm{ms}, i j}[E(B-V)]-m_{\mathrm{obs}, i}}{\delta m_{\mathrm{obs}, i}}\right\}^{2},
$$

where $N$ is the number of photometric points and $\delta m_{\mathrm{obs}, i}$ is the error on the observed magnitude $m_{\mathrm{obs}, i}$. The quantity $\delta m_{\mathrm{obs}, i}$, of course, is the sum in quadrature of the photometric error in the magnitude in band $i$ plus the one in the magnitude in the band to which the spectrum was normalized. The index $i$ in the sum runs on all bands but the one that was used to normalize the spectrum. If a star is not detected in a certain band, typically in the UV, a $3 \sigma$ upper limit for the flux is used. The models that predict a flux in that band higher than this limit are rejected.

Of course, the best-fit model corresponds to the combination of temperature and $E(B-V)$ that minimizes $\chi_{j}^{2}[E(B-V)]$.

4. Once the minimum $\chi_{j}^{2}$ is found, the errors in temperature $\left(\delta T_{\text {eff }}\right)$ and reddening $[\delta E(B-V)]$ are computed from the $\chi^{2}$ map.

5. Now that the shape of the spectrum is determined, we compute the radius of the star as the error-weighted mean of the logarithmic shifts required to match the model to the observed flux in every band. Since the theoretical fluxes are flux densities at the surface of the star, the angular radius, $\rho$, derived from band $i$ is

$$
\log \rho_{i}=-0.2\left(m_{i, \mathrm{obs}}^{0}-m_{\mathrm{ms}, i}^{\mathrm{fit}}\right),
$$

where $m_{\mathrm{ms}, i}^{\mathrm{fit}}$ is the magnitude in filter $i$ of the best-fitting theoretical spectrum and $m_{\mathrm{obs}, i}^{0}$ is the observed magnitude, dereddened with the best-fitting value of $E(B-V)$. The best estimate of the angular radius, $\rho$, then is

$$
\rho=\frac{\sum_{i=1}^{n}\left(\rho_{i} / \delta^{2} m_{\mathrm{obs}, i}\right)}{\sum_{i=1}^{n}\left(1 / \delta^{2} m_{\mathrm{obs}, i}\right)},
$$

where $n$ is the number of bands with actual flux measurements. The associated error, $\delta \rho$, then is

$$
(\delta \rho)^{2}=\frac{\sum_{i=1}^{n}\left(\rho_{i}-\rho\right)^{2} / \delta^{2} m_{\mathrm{obs}, i}}{(n-1) \sum_{i=1}^{n}\left(1 / \delta^{2} m_{\mathrm{obs}, i}\right)} .
$$

To convert the angular radius of a star to the linear one $(R)$, it is necessary to know its distance, $D$ :

$$
R=\rho \frac{D}{R_{\odot}} .
$$

In this case, $R$ is expressed in units of the solar radius, $R_{\odot}$.

Given the uncertainty on the distance, the error on the linear radius, $\delta R$, is

$$
\frac{\delta R}{R}=\sqrt{\left(\frac{\delta \rho}{\rho}\right)^{2}+\left(\frac{\delta D}{D}\right)^{2}} .
$$

This error does not take into account any intrinsic dispersion in the distance of individual stars, which may introduce additional scattering in the $\mathrm{H}-\mathrm{R}$ diagram.

For example, in the case of the LMC, we have adopted the value originally measured purely geometrically by Panagia et al. (1991) and later improved by Panagia (1999). 
Using the rings around SN 1987A, they found $D=$ $51.4 \pm 1.2 \mathrm{kpc}$.

6. Finally, the intrinsic luminosity $(L)$ can be computed according to the blackbody law:

$$
\frac{L}{L_{\odot}}=\left(\frac{R}{R_{\odot}}\right)^{2}\left(\frac{T_{\text {eff }}}{T_{\text {eff }, \odot}}\right)^{4},
$$

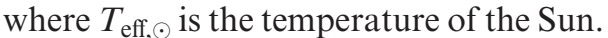

Since the errors in temperature and radius are correlated, the error on the luminosity cannot be computed in a straightforward way from the previous equation. It is more convenient to write the luminosity in another, completely equivalent form. Let us first define the quantities

$$
l_{\mathrm{obs}, i} \equiv 10^{-0.4\left(m_{\mathrm{obs}, i}^{0}-Z P_{i}\right)},
$$

i.e., the observed flux in filter $i$, and

$$
b \equiv \frac{\sigma T_{\mathrm{eff}}^{4}}{\sum_{i=1}^{n} l_{\mathrm{ms}, i}^{\mathrm{fit}}},
$$

where $l_{\mathrm{ms}, i}^{\mathrm{fit}}$ is the flux in filter $i$ of the best-fitting model. The quantity $b$ is the equivalent of the bolometric correction, relating the total flux in $n$ bands to the bolometric luminosity per unit surface, $\sigma T_{\text {eff }}^{4}$. We can now write the luminosity as

$$
L=4 \pi D^{2} l_{\mathrm{obs}} b,
$$

where $l_{\mathrm{obs}}=\sum_{i} l_{\mathrm{obs}, i}$. All the quantities in this last equation are independent of each other, and hence it is straightforward to compute the error on the luminosity:

$$
\frac{\delta L}{L}=\sqrt{\left(2 \frac{\delta D}{D}\right)^{2}+\left(\frac{\delta l_{\mathrm{obs}}}{l_{\mathrm{obs}}}\right)^{2}+\left(\frac{\delta b}{b}\right)^{2}}
$$

where

$$
\frac{\delta l_{\mathrm{obs}}}{l_{\mathrm{obs}}}=\frac{1}{l_{\mathrm{obs}}} \sqrt{\sum_{i=1}^{n}\left[\left(10^{0.4 \delta m_{\mathrm{ob}, i}}-1\right) l_{i, \mathrm{obs}}\right]^{2}}
$$

and

$$
\frac{\delta b}{b}=\frac{d b}{d T_{\text {eff }}} \delta T_{\text {eff }}
$$

In this last equation, it is clear that the first term on the right-hand side, $d b / d T_{\text {eff }}$, is completely determined by the models, whereas the second one, $\delta T_{\text {eff, }}$ is the error in the temperature from the fit computed in step 4 . In the case of the LMC, $\delta D / D=0.02$ (Panagia 1999).

Let us stress once again that the stars may not be all at the same distance and that this may introduce additional scattering in luminosity. From photometry alone, distances can be measured only in a statistical sense for an entire population and not for individual stars. However, since the intrinsic depth of the LMC is estimated to be smaller than 600 pc (Crotts, Kunkel, \& Heathcote 1995), this uncertainty is negligible.

\section{REFERENCES}

Allen, C. W. 1973, Astrophysical Quantities (3d ed.; London: Athlone) Arce, H. G., \& Goodman, A. A. 1999, ApJ, 512, L135

Bagget, S., Casertano, S., Gonzaga, S., \& Ritchie, C. 1997, WFPC2 SYNPHOT Update: (WFPC2 Instrument Science Report 97-10) (Baltimore: STScI)

Bagget, S., Sparks, W., Ritchie, C., \& MacKenty, J. 1996, Contamination Correction in SYNPHOT for WFPC2 and WF/PC-1 (WFPC2 Instrument Science Report 96-02) (Baltimore: STScI)

Bessel, M. S., Castelli, F., \& Plez, B. 1998, A\&A, 333, 231 (erratum A\&A, $337,321)$

Bianchi, L., Scuderi, S., Massey, P., \& Romaniello, M. 2001, AJ, 121, 2020

Biretta, J. A., et al. 2000, WFPC2 Instrument Handbook, Version 5.0 (Baltimore: STScI)

Brocato, E., \& Castellani, V. 1993, ApJ, 410, 99

Cassisi, S., Castellani, V., \& Straniero, O. 1994, A\&A, 282, 753

Castelli, F., Gratton, R. G.. \& Kurucz, R. L. 1997a, A\&A, 318, 841 1997b, A\&A, 324, 432

Crotts, A. P. S., Kunkel, W. E., \& Heathcote, S. R. 1995, ApJ, 438, 724

Fitzpatrick, E. L. 1988, ApJ, 335, 703

Gilliland, R. L. 1994, ApJ, 435, L63

Gilmozzi, R. 1990, Core Aperture Photometry with the WFPC (STScI Instrument Report WFPC-90-96) (Baltimore: STScI)

Kurucz, R. L. 1993, CD-ROM 13, ATLAS9 Stellar Atmosphere Programs and $2 \mathrm{~km} \mathrm{~s}^{-1}$ Grid (Cambridge: Smithsonian Astrophys. Obs.)

Mathis, J. S. 1990, ARA\&A, 28, 37
Mihalas, D., \& Binney, J. 1981, Galactic Astronomy: Structure and Kinematics (2d. ed.; San Francisco: Freeman)

Panagia, N. 1999, in IAU Symp. 190, New Views of the Magellanic Clouds, ed. Y.-H. Chu, N. Suntzeff, J. Hesser, \& D. Bohlender (San Francisco: ASP), 549

Panagia, N., Gilmozzi, R., Macchetto, F., Adorf, H. M., \& Kirshner, R. P. 1991, ApJ, 380, L23

Panagia, N., Romaniello, M., Scuderi, S., \& Kirshner, R. P. 2000, ApJ, 539,197

Romaniello, M. 1998, Ph.D. thesis, Scuola Normale Superiore (Pisa, Italy) Romaniello, M., Panagia, N., \& Scuderi, S. 2002, in preparation

Romaniello, M., Salaris, M., Cassisi, S., \& Panagia, N. 2000, ApJ, 530, 738

Savage, B. D., \& Mathis, J. S. 1979, ARA\&A, 17, 73

Schaerer, D., Meynet, G., Maeder, A., \& Schaller, G. 1993, A\&AS, 98, 523

Schlegel, D. J., Finkbeiner, D. P., \& Davis, M. 1998, ApJ, 500, 525

Schwering, P. B. W., \& Israel, F. P. 1991, A\&A, 246, 231

Scuderi, S., Panagia, N., Gilmozzi, R., Challis, P. M., \& Kirshner, R. P. 1996, ApJ, 465, 956

Walker, A. R., \& Suntzeff, N. B. 1990, PASP, 102, 131

Whitmore, B. 1995, in Calibrating the Hubble Space Telescope: Post Servicing Mission, ed. A. Koratkar \& C. Leitherer (Baltimore: STScI), 269

Whittet, D. C. B. 1992, Dust in the Galactic Environment (Bristol: Inst Physics)

Zaritsky, D. 1999, AJ, 118, 2824 